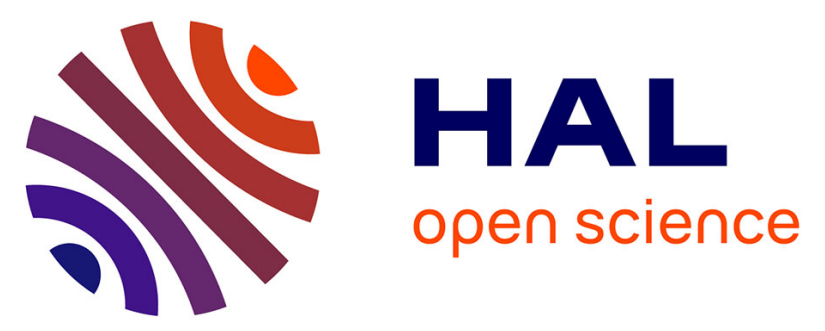

\title{
A Lagrangian Hencky-type non-linear model suitable for metamaterials design of shearable and extensible slender deformable bodies alternative to Timoshenko theory
}

Emilio Turco, Emilio Barchiesi, Ivan Giorgio, Francesco Dell'Isola

\section{- To cite this version:}

Emilio Turco, Emilio Barchiesi, Ivan Giorgio, Francesco Dell'Isola. A Lagrangian Hencky-type nonlinear model suitable for metamaterials design of shearable and extensible slender deformable bodies alternative to Timoshenko theory. International Journal of Non-Linear Mechanics, 2020, 123, pp.103481. 10.1016/j.ijnonlinmec.2020.103481 . hal-02914306

\author{
HAL Id: hal-02914306 \\ https://hal.science/hal-02914306
}

Submitted on 11 Aug 2020

HAL is a multi-disciplinary open access archive for the deposit and dissemination of scientific research documents, whether they are published or not. The documents may come from teaching and research institutions in France or abroad, or from public or private research centers.
L'archive ouverte pluridisciplinaire HAL, est destinée au dépôt et à la diffusion de documents scientifiques de niveau recherche, publiés ou non, émanant des établissements d'enseignement et de recherche français ou étrangers, des laboratoires publics ou privés. 


\title{
A Lagrangian Hencky-type non-linear model suitable for metamaterials design of shearable and extensible slender deformable bodies alternative to Timoshenko theory
}

\author{
Emilio Turco $^{\text {a }}$, Emilio Barchiesi ${ }^{\mathrm{b}}$, Ivan Giorgio ${ }^{\mathrm{c}}$, Francesco dell'Isola ${ }^{\mathrm{d}}$ \\ a Department of Architecture, Design and Urban planning (DADU), University of Sassari, Italy \\ ${ }^{\mathrm{b}}$ MEMOCS, International Research Center for Mathematics and Mechanics of Complex Systems, University of L'Aquila, Italy \\ ${ }^{\mathrm{c}}$ Department of Civil, Construction-Architectural and Environmental Engineering, University of L'Aquila, Italy \\ 'Department of Structural and Geotechnical Engineering, University of Rome "La Sapienza", Italy
}

\begin{abstract}
A B S T R A C T
Among the most studied models in mathematical physics, Timoshenko beam is outstanding for its importance in technological applications. Therefore it has been extensively studied and many discretizations have been proposed to allow its use in the most disparate contexts. However, it seems to us that available discretization schemes present some drawbacks when considering large deformation regimes. We believe these drawbacks to be mainly related to the fact that they are formulated without keeping in mind the mechanical phenomena for describing which Timoshenko continuum model has been proposed. Therefore, aiming to analyze the deformation of complex plane frames and arches in elastic large displacements and deformation regimes, a novel intrinsically discrete Lagrangian model is here introduced whose phenomenological application range is similar to that for which Timoshenko beam has been conceived. While being largely inspired by the ideas outlined by Hencky in his renowned doctoral dissertation, the presented approach overcomes some specific limitations concerning the stretch and shear deformation effects. The proposed model is applied to get the solutions for some relevant benchmark tests, both in the case of arch and frame structures. It is proved that, also when shear deformation effects are of relevance, the enriched, yet simple, model and numerical computation scheme herein proposed can be profitably used for efficient structural analyses of non-linear mechanical systems in rather nonstandard situations.
\end{abstract}

\section{Introduction}

Since the fundamental works by Euler and Bernoulli, the phenomenology of the deformation of slender bodies has been described using the so-called reduced continuum models, like Euler-Bernoulli beam, see, e.g., [1] for a general introduction to beam models and their relations (Timoshenko model approaches to the Euler-Bernoulli model when the beam thickness approaches to zero with respect to certain mathematical measures, see also [2] for plate models). The fundamental idea of reduced models, in this context, is simple, and has been traced back to Galileo and Leonardo by Benvenuto [3]. Indeed, in the literature, it is widely accepted that in Galileo's works one finds traces of an attempt to develop a form of reduced model for the deformation of slender bodies, i.e. what is usually called a version of a theory of beam.

On the other hand such an attempt has been recognized also in some manuscripts by Leonardo (see [4]) even if Leonardo's originality, competence and knowledge in mechanics has been bitterly disputed (see [5]). The important observations found in Leonardo's manuscript did not manage to give an efficient picture of the phenomenology, as one does not find in it any trace of elastic constitutive laws or any form of differential calculus in his treatment, even if a kind of one-dimensional model is sketched. On the other hand, also Galileo's theory is flawed by some wrong assumptions about the beams' section deformation modalities. There is the possibility that Leonardo, as he did in other situations, based his considerations on more ancient sources.

In facts, a reduced model deduces (or postulates) a deformation modality in the sections of the considered slender deformable body by introducing displacement and deformation fields depending only on the curvilinear abscissa along its axis. The first modern beam theory is usually attributed to Jacob and Daniel Bernoulli, together with Euler, even if the crucial results in Calculus of Variations which are needed in the model development must be credited to Lagrange. Bernoulli-Euler beam theory represents one of the first scientific models successfully 


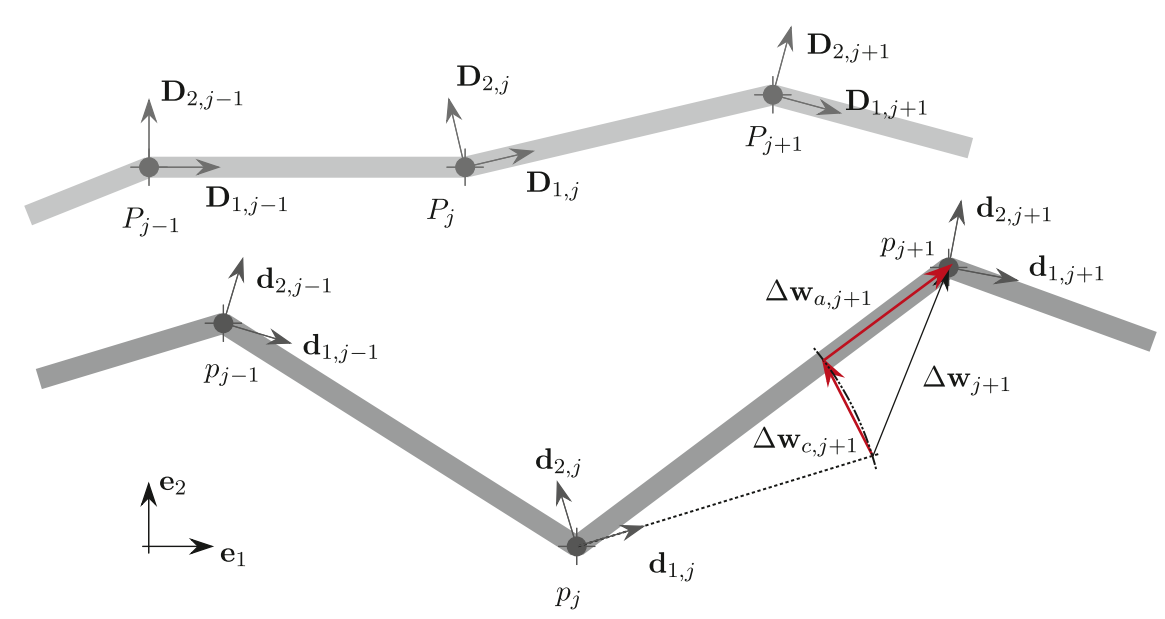

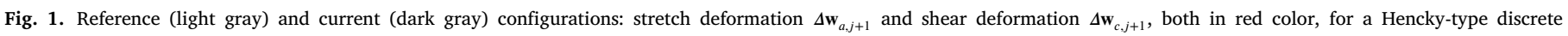
Timoshenko beam in plane.

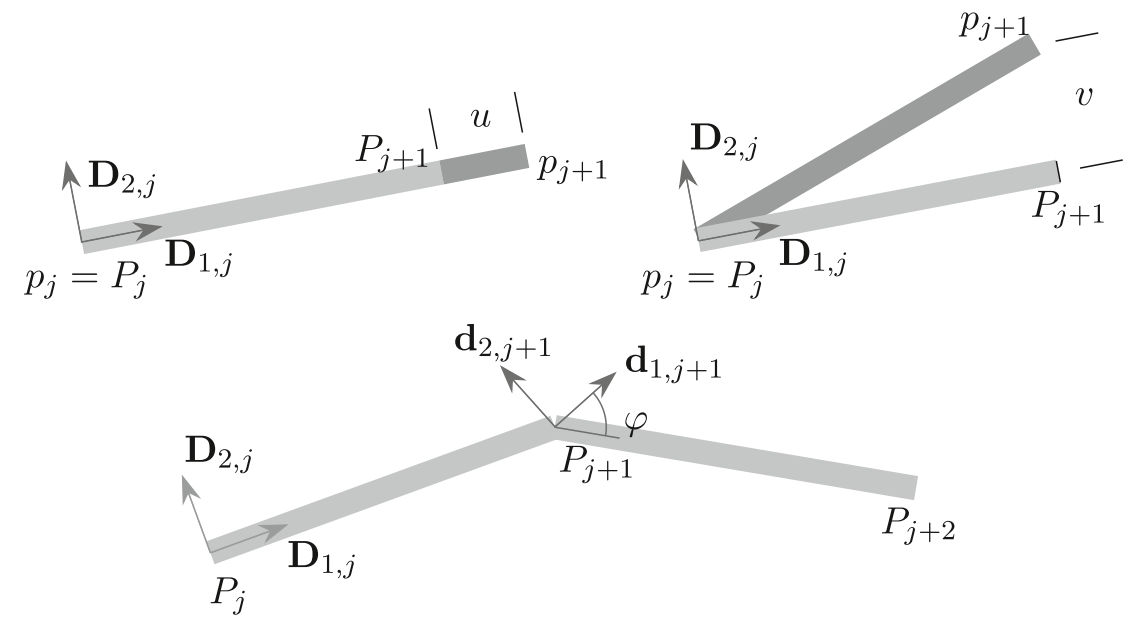

Fig. 2. Superimposed deformation modes. First test (left-top), second test (right-top), and third test (bottom).

applied to engineering and technology design, proving that mathematical models could be safely applied in the applications.

However, reduced models fail to describe some important deformation phenomena: de Saint-Venant did apply Cauchy 3D continuum models to describe some of them, as anticlastic curvature, shear deformation or warping. However, Timoshenko ${ }^{1}$ did manage to improve Euler-Bernoulli theory by using, instead of a more detailed 3D model, still a 1D continuum model which considers an extra kinematical descriptor, i.e. the descriptor of section rotation. Among the most studied models in mathematical physics, Timoshenko beam is outstanding for its importance in technological applications: for instance it allowed for the safe design of airplane wings.

Timoshenko beam model is richer than Euler-Bernoulli beam model, as the cross-section rotation is a kinematic descriptor independent of the displacement field. This choice supplies us a more predictive model, when compared with Euler-Bernoulli beam, however at the price of being more expensive computationally. On the other hand it is less descriptive and less computationally expensive when compared with 3D continuum models: it can be a good compromise in several situations.

Therefore, it has been studied by scholars in many different fields of mechanics and related subjects: applied mathematics [7], structural mechanics (in both static [8] and dynamic [9,10] problems), and computational mechanics [11-14], just to name a few. The abounding deal

1 According to Elishakoff [6], one should write Timoshenko and Ehrenfest. of literature about Timoshenko beam theory is an evident proof of its importance: it is traditionally exploited in all structural problems where a description of slender shear deformable structural elements is needed. It is often considered also as a prototype example in the theory of media with micro-strains. In addition, the interest for the use of Timoshenko model in the mechanical analysis of metamaterials has exponentially growth in the last year. In fact, very often the internal structure of mechanical metamaterials requires the exploitation of beam models with a richer kinematics than that of the Euler-Bernoulli beam model, see, e.g., [15-19]. As Timoshenko beam is a continuous model, a discretization is necessary in order to get predictions by numerical codes, see, e.g., [20,21]. However, many different schemes among those available present relevant drawbacks, especially when considering the case of large deformations regimes: most likely these drawbacks are mainly related to the fact that usually discretization schemes are formulated mathematically or, in general, without explicitly considering the true nature of mechanical phenomena whose description motivated the formulation of Timoshenko continuum model, see, e.g., [22] for a classic work and [21] for a more recent one.

In this paper, as we intend to start the construction of a tool aimed to analyze the large elastic deformation phenomena of complex plane frames and arches, a novel intrinsically discrete Lagrangian model is directly postulated with a twofold aim: (i) to supply an effective model having a phenomenological application range similar to that for which continuum Timoshenko beam has been conceived and (ii) to take into account in a detailed and judicious way the true nature of the considered mechanical systems. 


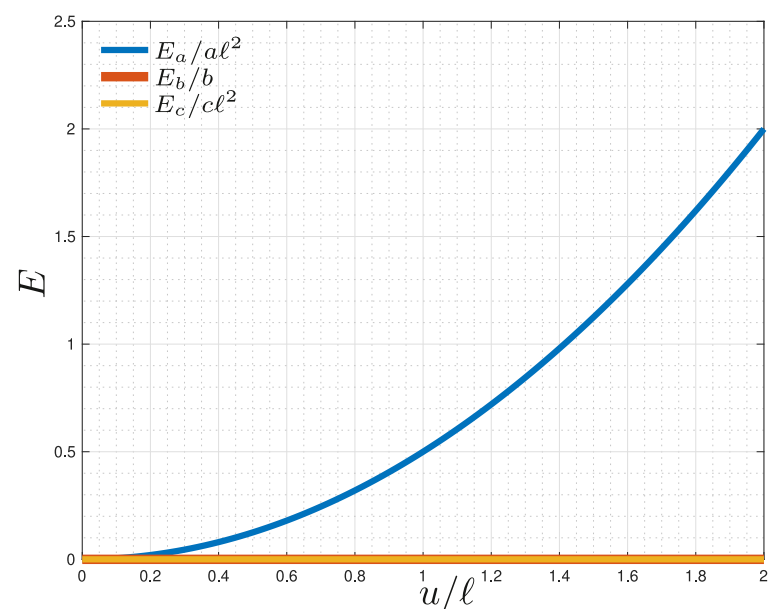

(a) First test.

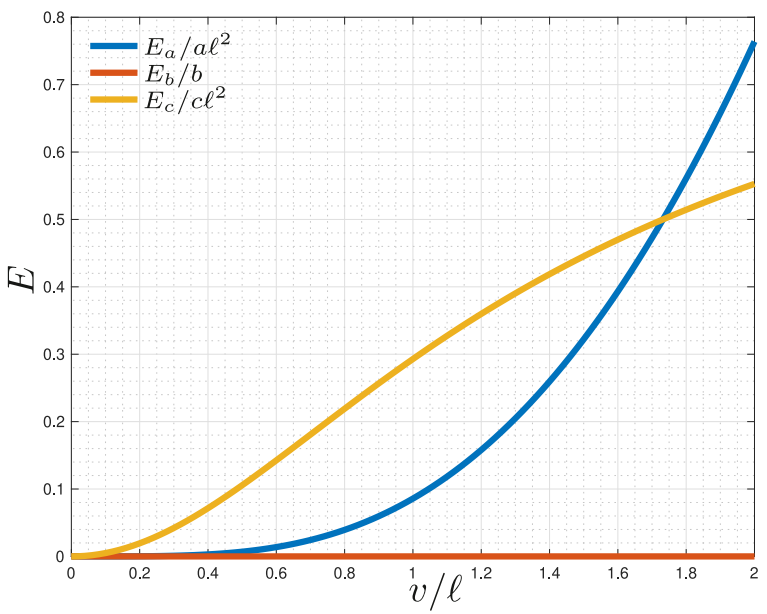

(b) Second test.

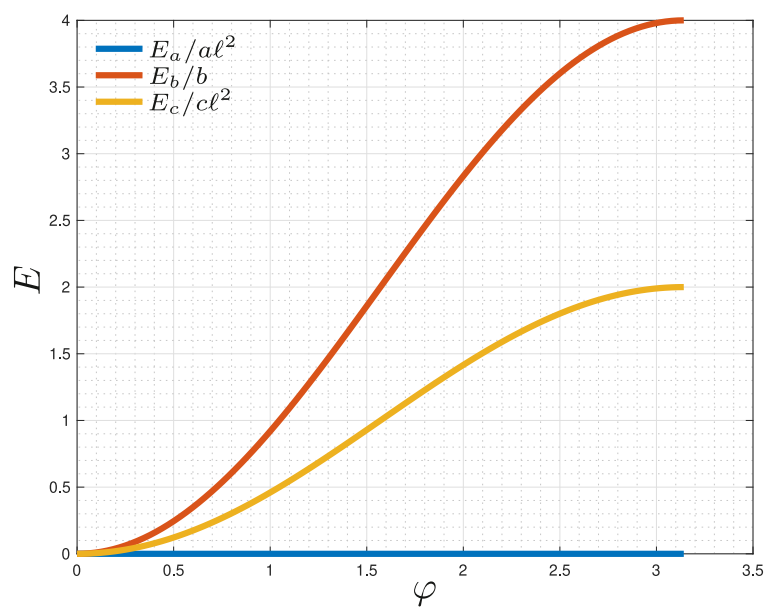

(c) Third test.

Fig. 3. Strain energy as split into scaled stretch, bending and shear contributions for tests 1,2 and 3 against $u / \ell, v / \ell$, and $\varphi$, respectively.

The presented intrinsically discrete model is clearly and largely inspired by the ideas presented by Hencky in his doctoral dissertation: however it is general enough to be able to describe also the most important stretch and shear deformation effects. In this paper, the

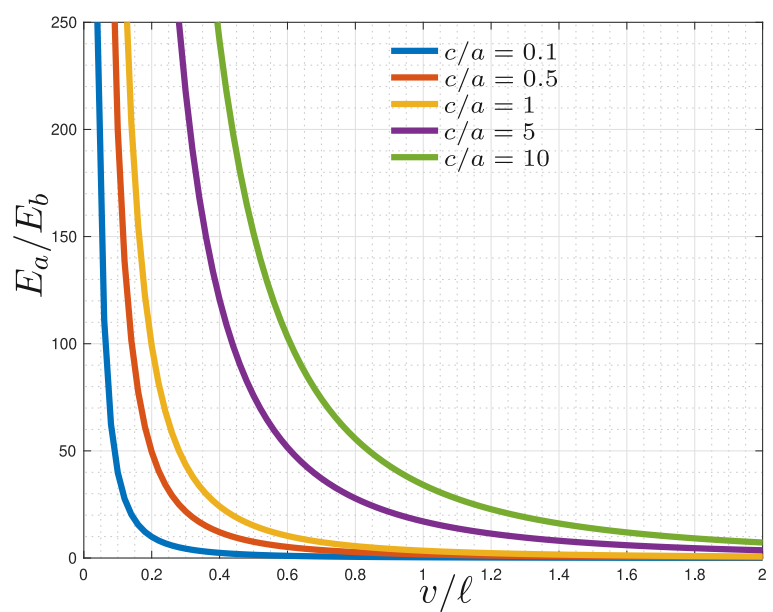

Fig. 4. Shear-to-stretch strain energy ratio versus the dimensionless displacement $v / \ell$ for the second test as the ratio $c / a$ from 0.1 to 10 varies.

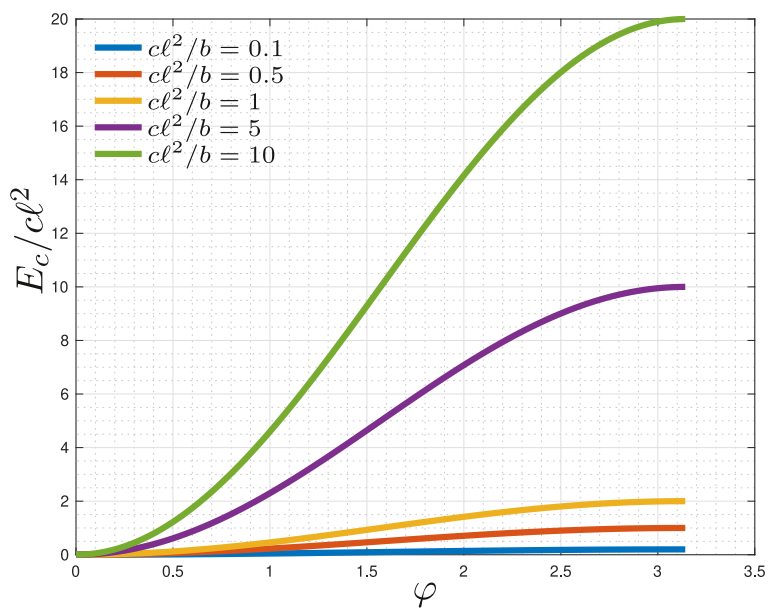

Fig. 5. Dimensionless shear strain energy $E_{c} / c \ell^{2}$ versus $\varphi$ for the third test as the non-dimensional stiffness ratio $c \ell^{2} / b$ varies from 0.1 to 10 .

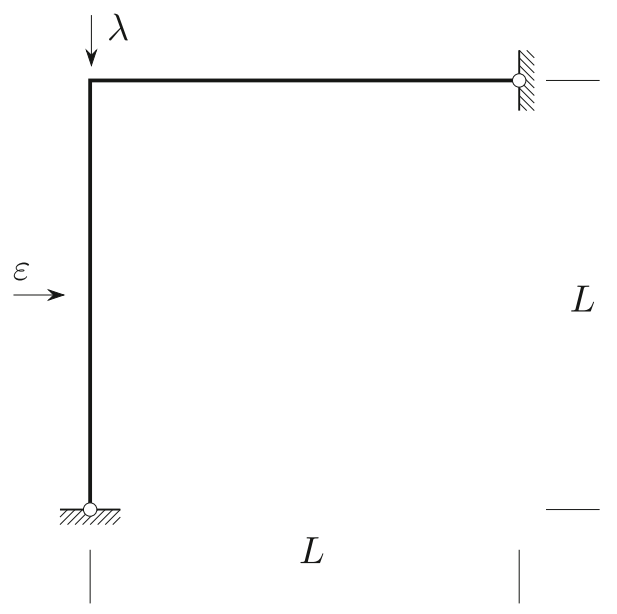

Fig. 6. Roorda's frame.

postulated Hencky-type model is used to show how it allows for the correct determination of the solutions of some relevant benchmark tests where both arches and frames are involved. We additionally prove that, also when shear deformation effects are to be described, the 


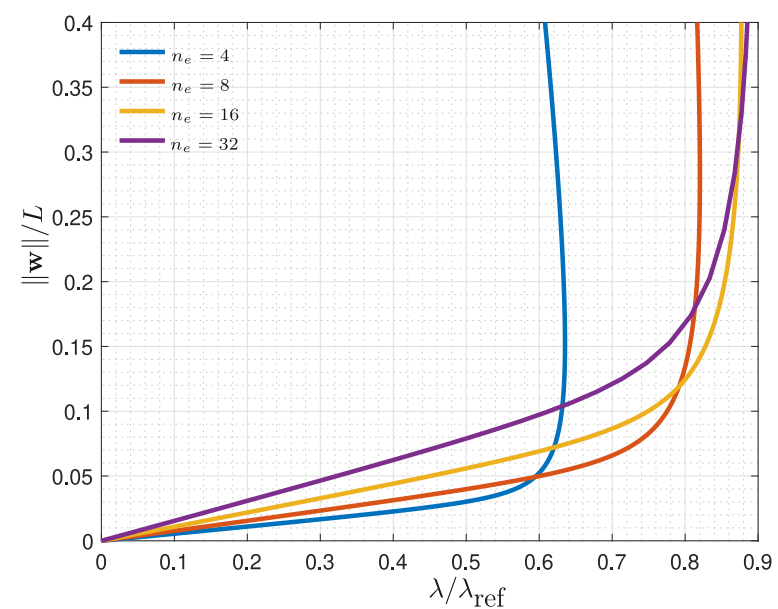

Fig. 7. Roorda's frame: equilibrium paths obtained by increasing the number of discrete elements $n$ utilized to model the beams forming the frame $\left(a L^{2} / b=1000, c L^{2} / b=1000\right.$ and $\varepsilon=0.0001$ ).

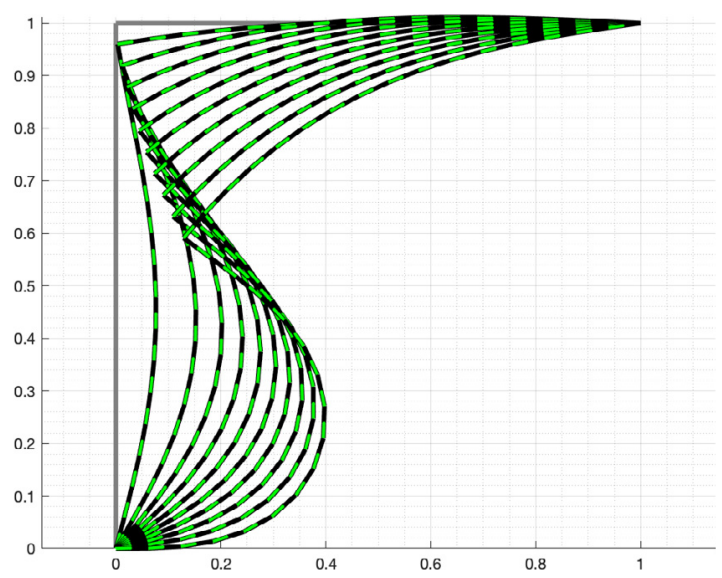

Fig. 8. Roorda's frame: evolution of deformed configuration for subsequent load steps $\left(n=32, a L^{2} / b=1000, c L^{2} / b=1000, \varepsilon=0.0001\right)$.

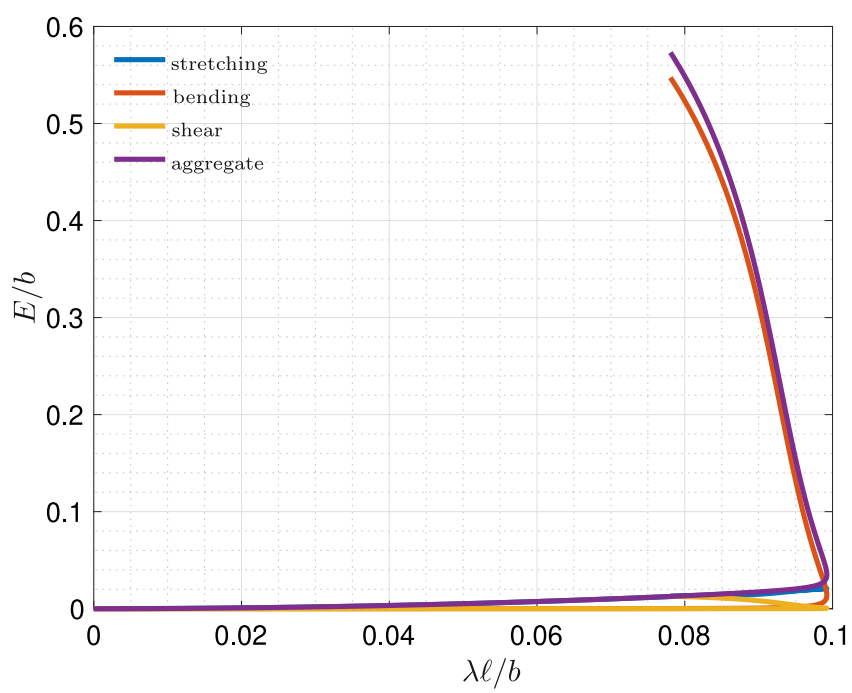

Fig. 9. Roorda's frame: dimensionless energy for subsequent load steps as split in stretch, bending and shear contributions $\left(n_{e}=32, a L^{2} / b=100, c L^{2} / b=1, \varepsilon=0.001\right)$.

proposed discrete model is very efficient in describing the deformation of non-linear mechanical structures in nonstandard situations.

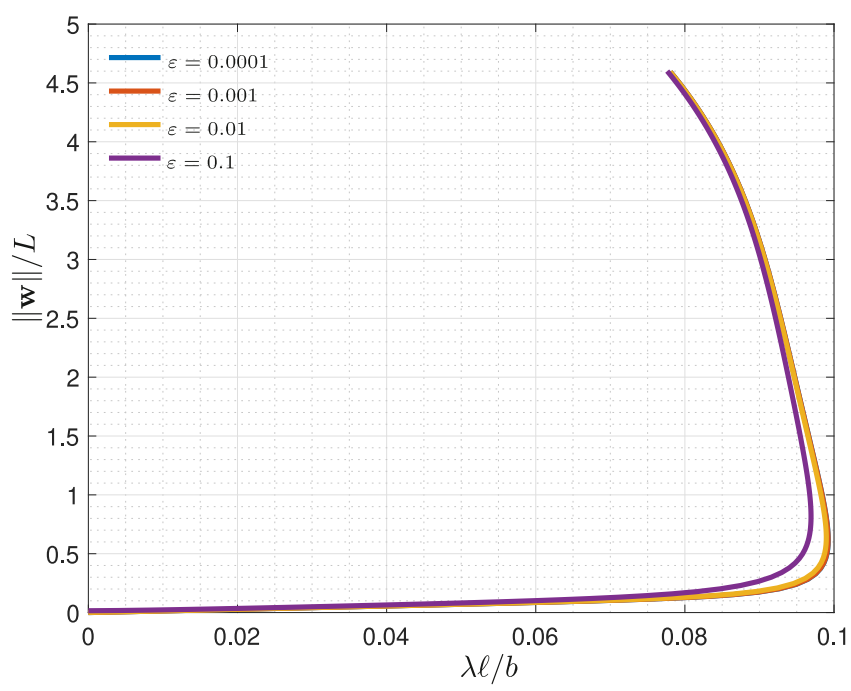

Fig. 10. Roorda's frame: equilibrium paths obtained with $n=32$ discrete elements by increasing the imperfection magnitude $\varepsilon\left(a L^{2} / b=1000, c L^{2} / b=1000\right)$.

As in the literature the existence of multiple solutions to the problem of large deformations of structures in presence of dead loads has been considered a serious and difficult challenge, from both the numerical and analytical points of view, we apply the proposed discrete model (as implemented in a numerical computation scheme) to find the two stable deformation shapes of a cantilever beam under a vertical dead load. This simple structure has represented a challenge which caused the failure of many other numerical schemes and supplied interesting problems in the field of direct calculus of variations (see [23]).

The proposed model and numerical scheme have easily allowed for the computation of both said stable configurations, simply by suitably changing the two introduced dead load parameters: it is therefore reasonable to conjecture that they can be successfully exploited in the design and study of those novel mechanical metamaterials which are constituted, at a suitable mesoscopic length-scale, by a system of complex beam lattices.

In this context, we recall the study of so-called pantographic metamaterials in [24,25]: the slender elements constituting the pantographic lattice might have axial length and/or cross-section dimensions not allowing the use of Euler-Bernoulli beam model, i.e. the use of a model which is neglecting a priori the shear deformation phenomena. Indeed, there is no reason to ignore the contribution of shear deformation without a preliminary analysis aimed at defining its influence. Moreover one may be willing to exploit shear deformation effects in the design of some novel metamaterials: this research perspective is rather promising as the analyses by discrete mesomechanical modeling of lattice structures have been often exploited with remarkably good results for the design of complex metamaterials, where more detailed modeling is not amenable to current computational capabilities.

In facts this idea was exploited already in [26], whose aim was to determine an evaluation of the buckling load for an inextensible EulerBernoulli beam in large deformations by using a discrete formulation: Hencky limited his treatment to the case of large deformations of an Elastica, i.e. a beam model where only the flexural deformability is considered. Hencky procedure has been later firmly mathematically based, as it is well established now that in the limit for vanishing characteristic length of a discrete mesomechanical model many discrete models approximate a corresponding homogenized continuum. On the other hand, often, such a continuum model is then again discretized without any reference to the underlying micro-structure when numerical solutions are needed by, e.g., a finite element analysis. When amenable to computations, it seems to us that it is less intricate to solve numerically directly the problem for the mesomechanical model. This 


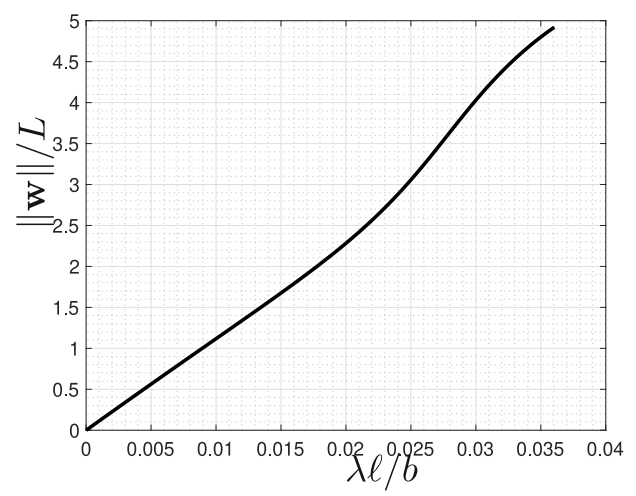

(a)

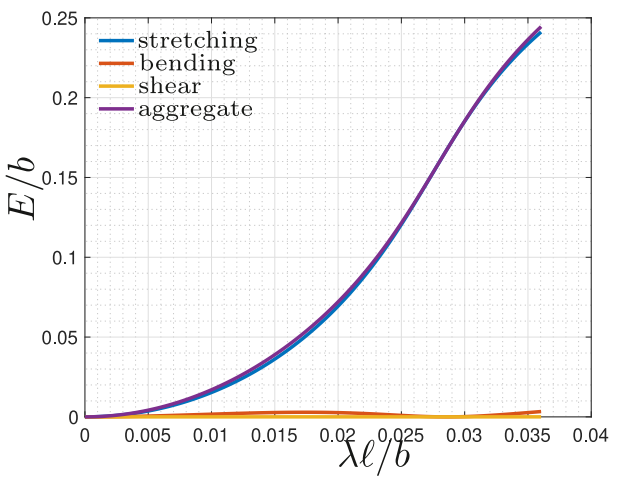

(c)

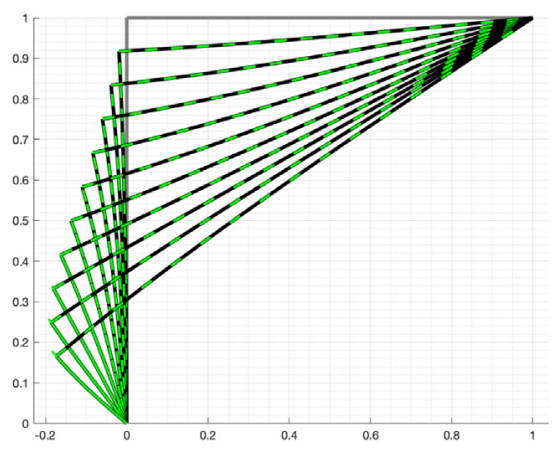

(e)

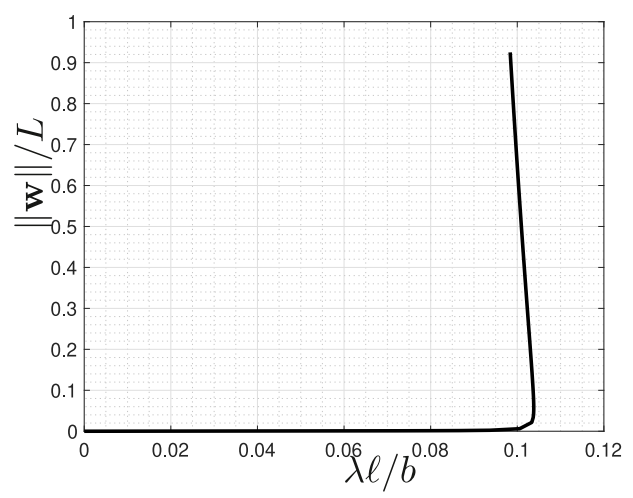

(b)

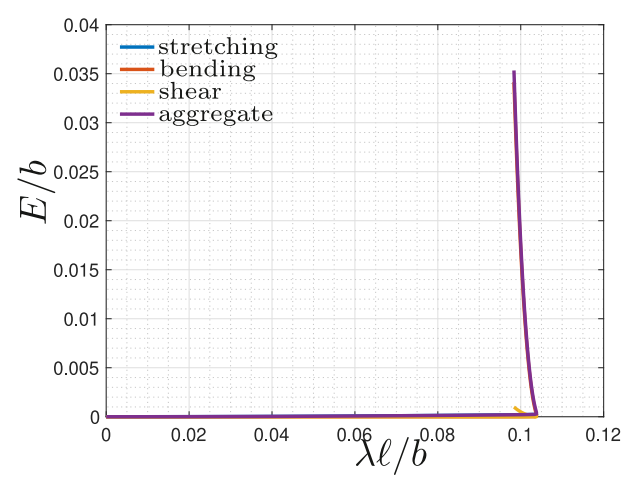

(d)

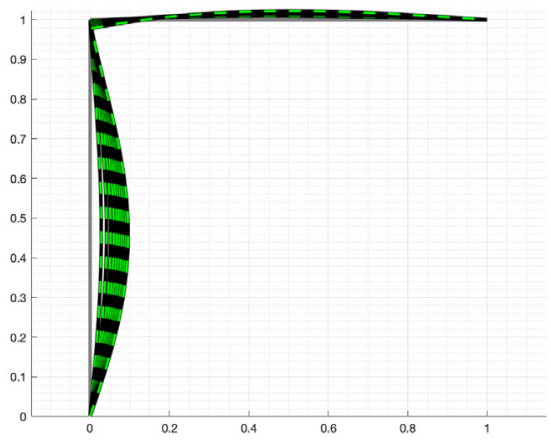

(f)

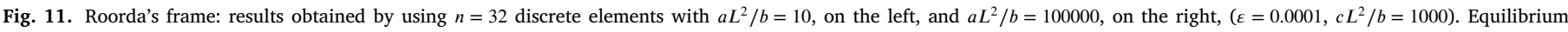
path (1st row), evolution of non-dimensional strain energy contributions (2nd row) and of deformed configuration (3rd row) for subsequent load steps.

kind of analysis has been proved to be very effective in predicting the nonlinear mechanical behavior of complex materials.

Based on the previous considerations, we present in this article a discrete mesomechanical beam formulation, see also [27,28], which takes into account not only beam stretch, i.e. avoiding the inextensibility hypothesis, but also shear deformability. Subsequent developments of the present papers will involve the generalization of the works [2931]. In these works the bending strain is defined for beams moving in the three-dimensional space, while here for the sake of simplicity we limit our attention to in-plane beams. Anyway, the formulation of the present discrete model can be easily transferred to beams moving in a three-dimensional space, see $[32,33]$.

The plan of the paper is the following. In Section 2, the proposed discrete Hencky-type formulation of in-plane Timoshenko beam is given by defining its kinematics and strain energy. Successively, both the structural reaction, i.e. the gradient of strain energy with respect to the Lagrangian parameters, and the stiffness matrix, i.e. its Hessian with respect to the Lagrangian parameters are introduced. These last quantities are the main ingredients used in Section 3 to implement a path-following solution strategy using a method largely inspired to the Riks' arc-length method [34]. In Section 4 there is a brief discussion 50 about the influence of the shear deformability and in Section 5 the proposed model is solved in relevant benchmark tests for arch and frame structures and the influence of the shear stiffness is investigated. Finally, some concluding remarks and future challenges are discussed in Section 6.

\section{Modeling of two-dimensional Timoshenko beams}

We consider a planar beam, not necessarily rectilinear, made up by the finite arrangement of straight links connected by joints, that are sketched in Fig. 1 as gray circles. 


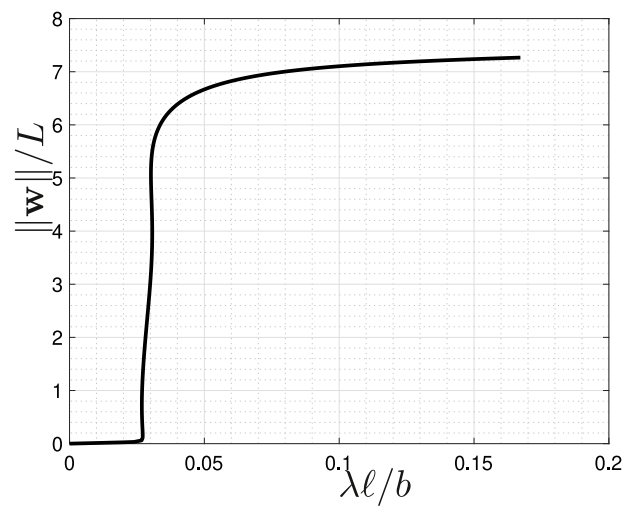

(a)

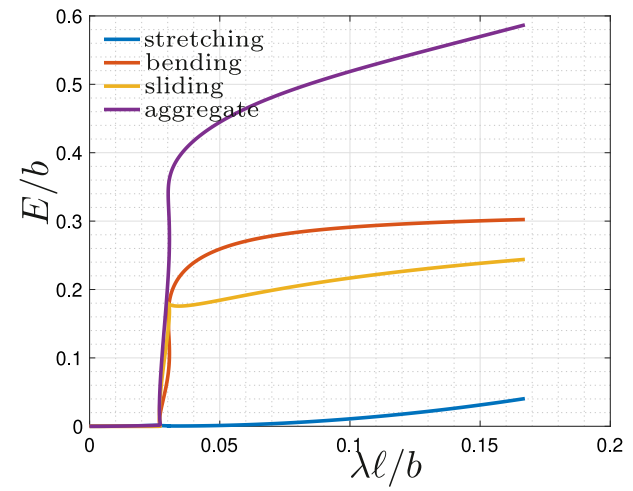

(c)

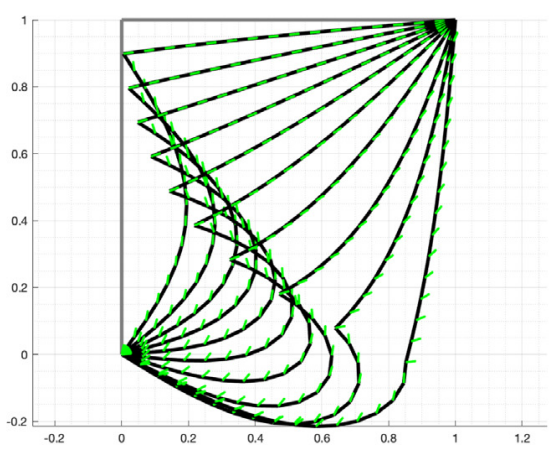

(e)

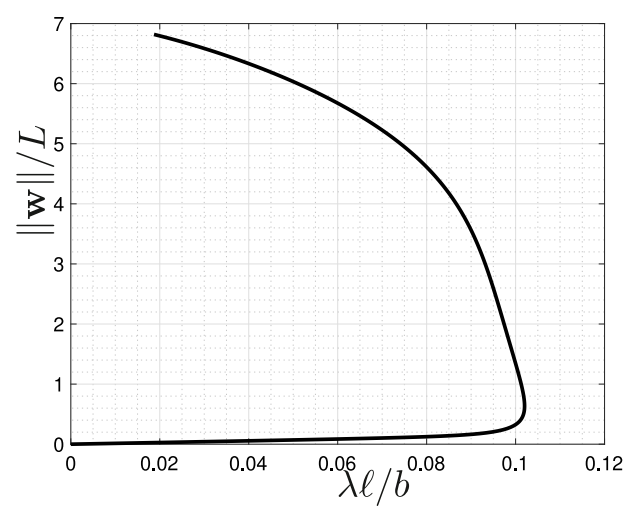

(b)

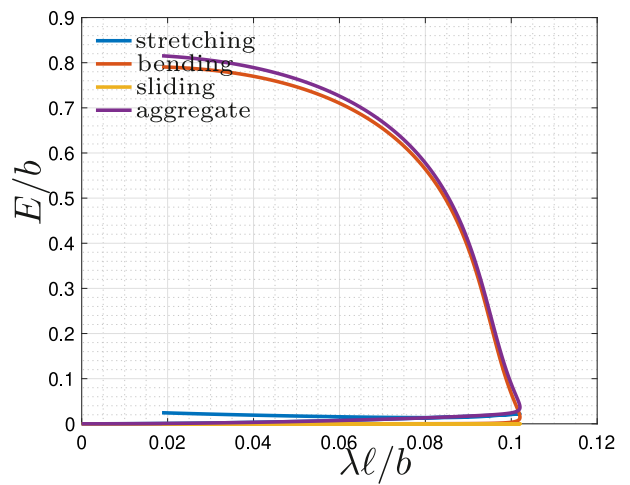

(d)

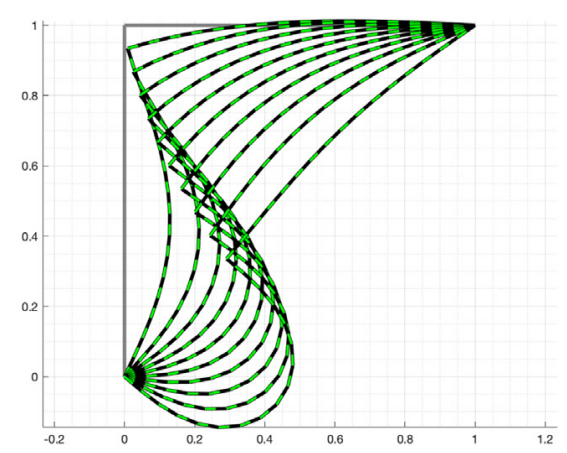

(f)

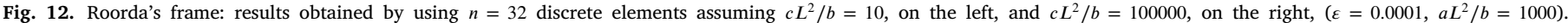
Equilibrium path (1st row), evolution of non-dimensional strain energy contributions (2nd row) and of deformed configuration (3rd row) for subsequent load steps.

The positions of the $j$ th joint in the reference and current configurations are $P_{j}$ and $p_{j}$, respectively, with $j=1,2, \ldots, N$. Exploiting the same rational of polar media continuum mechanics, each joint is also equipped, in the reference configuration, with a unit vector $\mathbf{D}_{1, j}=$ $\frac{P_{j+1}-P_{j}}{\left\|P_{j+1}-P_{j}\right\|}$. Although it is not mandatory in the two-dimensional case, in order to extend in the next future the Hencky-type beam element presented here, we consider for each joint a right-hand orthogonal triad $\left\{\mathbf{D}_{1, j}, \mathbf{D}_{2, j}, \mathbf{D}_{3, j}\right\}$, where $\mathbf{D}_{3, j}=\mathbf{D}_{1, j} \times \mathbf{D}_{2, j}$.

The orthogonal 3-tuple $\left\{\mathbf{D}_{1, j}, \mathbf{D}_{2, j}, \mathbf{D}_{3, j}\right\}$ is transformed in the current configuration into the 3-tuple $\left\{\mathbf{d}_{1, j}=\mathbf{Q}_{j} \mathbf{D}_{1, j}, \mathbf{d}_{2, j}=\mathbf{Q}_{j} \mathbf{D}_{2, j}, \mathbf{d}_{3, j}=\right.$ $\mathbf{Q}_{j} \mathbf{D}_{3, j}$, being $\mathbf{Q}_{j}$ a proper orthogonal second-rank rotation tensor.

Reference and current configurations are hence described by the following pairs of Lagrangian parameters $\left\{P_{j}, \mathbf{D}_{1, j}\right\}$ and $\left\{p_{j}, \mathbf{d}_{1, j}\right\}$, respectively.
Making reference to Fig. 1, we define as strain measures the vector $\Delta \mathbf{w}_{j+1}$ given by

$\Delta \mathbf{w}_{j+1}=\left(p_{j+1}-p_{j}\right)-\left\|P_{j+1}-P_{j}\right\| \mathbf{Q}_{j} \mathbf{D}_{1, j}$,

and the tensor $\Delta \mathbf{Q}_{j+1}$ given by

$\Delta \mathbf{Q}_{j+1}=\mathbf{Q}_{j}^{T} \mathbf{Q}_{j+1}-\mathbf{I}$,

with I being the identity second-rank tensor. The vector $\Delta \mathbf{w}_{j+1}$ is the difference between $p_{j+1}-p_{j}$ and $\left\|P_{j+1}-P_{j}\right\| \mathbf{Q}_{j} \mathbf{D}_{1, j}$. The former is the vector connecting the $j$ th and $(j+1)$-th nodes in the current configuration, while the latter is the vector having norm $\left\|P_{j+1}-P_{j}\right\|$ and obtained rotating $\mathbf{D}_{1, j}$ by $\mathbf{Q}_{j}$. The strain vector $\Delta \mathbf{w}_{j+1}$ can be sub-divided additively as

$\Delta \mathbf{w}_{a, j+1}=\left(p_{j+i}-p_{j}\right)\left(1-\frac{\left\|P_{j+1}-P_{j}\right\|}{\left\|p_{j+1}-p_{j}\right\|}\right)$, 


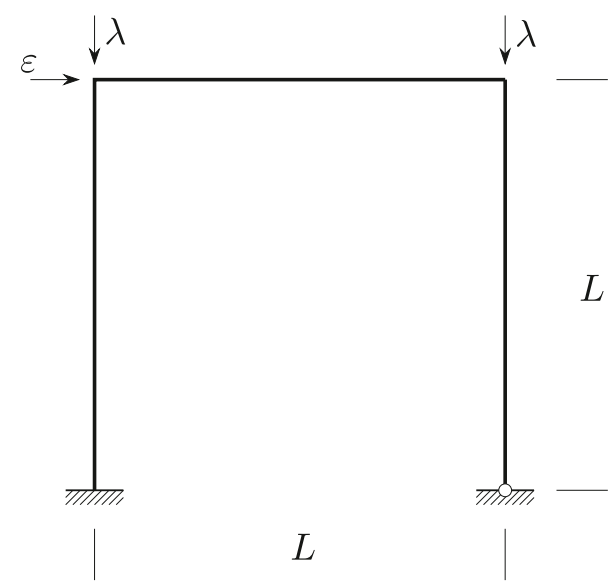

Fig. 13. Hinged-hinged frame under compression loads and horizontal imperfection load.

$\Delta \mathbf{w}_{c, j+1}=\Delta \mathbf{w}_{j+1}-\Delta \mathbf{w}_{a, j+1}$,

being the graphic representation of each quantity above sketched in Fig. 1.

The norms of the two vectors in (3)-(4) describe stretch and shear deformations, respectively, of the link in-between the $j$ th and $(j+1)$-th joints.

The tensor $\Delta \mathbf{Q}_{j+1}$ is a measure of beam bending, which is defined in the present discrete formulation as the relative rotation between the two adjacent links concurring at node $j+1$. The quantity $\Delta \mathbf{Q}_{j+1}+$ $\mathbf{I}=\mathbf{Q}_{j}^{T} \mathbf{Q}_{j+1}$ is a proper orthogonal tensor. When $\mathbf{Q}_{j}=\mathbf{Q}_{j+1}$ then $\Delta \mathbf{Q}_{j+1}$ is the null tensor. As done in [33] for the discrete formulation of an inextensible Euler-Bernoulli beams moving in space, owing to Rodrigues' formula [35], a rotation of amplitude $\varphi$ about the unitary axis e can be represented by the proper orthogonal tensor $\mathbf{Q}$ given by

$\mathbf{Q}=\cos \varphi \mathbf{I}+(1-\cos \varphi) \mathbf{e} \otimes \mathbf{e}+\sin \varphi \mathbf{E}$,

where $\mathbf{E}$ denotes the skew tensor whose axial vector is $\mathbf{e}$, i.e. $\mathbf{E u}=\mathbf{e} \times \mathbf{u}$. Eq. (5) expresses the rotation tensor in terms of the rotation angle $\varphi$ and the rotation axis e. In two-dimensional motions the kinematics simplifies since the rotation axis $\mathbf{e}$ is always directed along the unit vector $\mathbf{e}_{3}=\mathbf{e}_{1} \times \mathbf{e}_{2}$, see again Fig. 1, it being $\left\{\mathbf{e}_{1}, \mathbf{e}_{2}, \mathbf{e}_{3}\right\}$ a triad of orthogonal unit vectors.

For example, the skew tensor $\mathbf{E}$ and the rotation tensor $\mathbf{Q}$, as expressed in the vector basis $\left\{\mathbf{e}_{1}, \mathbf{e}_{2}, \mathbf{e}_{3}\right\}$, become

$\mathbf{E}=\left[\begin{array}{ccc}0 & -1 & 0 \\ 1 & 0 & 0 \\ 0 & 0 & 0\end{array}\right]$

and

$\mathbf{Q}=\left[\begin{array}{ccc}\cos \varphi & -\sin \varphi & 0 \\ \sin \varphi & \cos \varphi & 0 \\ 0 & 0 & 1\end{array}\right]$,

respectively.

So far, we have described the relationships between the independent Lagrangian parameters used to describe the motion, i.e. displacements of joints and rotations of the links, and the chosen strain measures $\Delta \mathbf{w}_{a, j}, \Delta \mathbf{w}_{c, j}$ and $\Delta \mathbf{Q}_{j+1}$. At this point, we define the strain energy of the whole beam as the summation of the following elementary contributions

$$
\begin{aligned}
& 2 E_{a}=a\left\|\Delta \mathbf{w}_{a, j+1}\right\|^{2}, \\
& 2 E_{b}=b\left\|\Delta \mathbf{Q}_{j+1}\right\|^{2}, \\
& 2 E_{c}=c\left\|\Delta \mathbf{w}_{c, j+1}\right\|^{2},
\end{aligned}
$$

where $\left\|\Delta \mathbf{w}_{a, j+1}\right\|$ and $\left\|\Delta \mathbf{w}_{c, j+1}\right\|$ are the Euclidean norms of the vectors $\Delta \mathbf{w}_{a, j+1}$ and $\Delta \mathbf{w}_{c, j+1}$, respectively, $\left\|\Delta \mathbf{Q}_{j+1}\right\|^{2}=\operatorname{tr}\left(\Delta \mathbf{Q}_{j+1}^{T} \Delta \mathbf{Q}_{j+1}\right)$, and $a$, $b$, and $c$ are stiffness parameters corresponding to stretch, bending and shear deformations of the beam, respectively.

Remark that when $\mathbf{d}_{j}$ is required to be parallel to the segment connecting the $j$ th and $(j+1)$-th nodes, then there is no shear deformation and one retrieves the Hencky approximation of the non-linear inextensible Euler-Bernoulli model as described, e.g., in the work [36].

In what follows, the one-dimensional continuum limit of the proposed discrete system will be derived for arbitrarily large deformations by means of a heuristic asymptotic homogenization argument. The total size of the system will be kept fixed in the homogenization process, while the number of the cells will be let tending to infinite. The continuum limit will be then specialized to the small-strain case and Timoshenko beam will be recovered, showing that the proposed formulation is a novel discrete non-linear generalization of Timoshenko model. For simplicity, in the undeformed configuration, cells are considered to be arranged upon a straight line in direction $\mathbf{D}_{1}$. Furthermore, the quantity $\left\|P_{j+1}-P_{j}\right\|=\ell$ will be assumed to be independent of the index $j$. The target one-dimensional continuum, to be obtained in the limit of vanishing $\ell$, is considered as parametrized by the arc-length $s \in[0, L]$ of the straight segment of length $L$ connecting all points $P_{j}$. The independent kinematic Lagrangian descriptors of the target continuum macro-model are assumed to be the functions

$\chi:[0, L] \rightarrow \mathbb{E}^{2}, \quad \phi:[0, L] \rightarrow[0,2 \pi)$.

The function $\chi$ places the $1 \mathrm{D}$-continuum into $\mathbb{E}^{2}$ and is intended to describe the points $p_{j} \in \mathbb{E}^{2}$ of the discrete system on a macro-level, while the function $\phi$ is meant to describe on a macro-level the rotation of unit vectors $\mathbf{D}_{1, j}$. In the sequel, prime will denote differentiation with respect to the reference arc length $s$. Owing to notational convenience, the quantity $\mathbf{Q}_{j} \mathbf{D}_{1, j}$, which is equal to $\mathbf{d}_{1, j}$, will be henceforth denoted with $\mathbf{e}\left(\phi_{j}\right)$. By Piola's ansatz, generalized coordinates of the discrete system are related with the functions (11) evaluated at $s_{j}=j h$ according to the following relations

$\chi\left(s_{j}\right)=p_{j}, \quad \phi\left(s_{j}\right)=\phi_{j}$

where $\phi_{j}$ is the rotation amplitude associated to the rotation tensor $\mathbf{Q}_{j}$. The energies (8)-(10) shall now be expanded with respect to $\ell$. Preliminarily, it is remarked that $\operatorname{tr}\left(\Delta \mathbf{Q}_{j+1}^{T} \Delta \mathbf{Q}_{j+1}\right)=\operatorname{tr}\left[\left(\mathbf{Q}_{j}^{T} \mathbf{Q}_{j+1}-\right.\right.$ $\left.I)^{T}\left(\mathbf{Q}_{j}^{T} \mathbf{Q}_{j+1}-I\right)\right]$ can be written in terms of $\varphi_{j}$ as

$$
\begin{aligned}
\operatorname{tr}\left(\Delta \mathbf{Q}_{j+1}^{T} \Delta \mathbf{Q}_{j+1}\right) & =2\left\{\left[\cos \left(\phi_{j}\right) \sin \left(\phi_{j+1}\right)-\sin \left(\phi_{j}\right) \cos \left(\phi_{j+1}\right)\right]^{2}\right. \\
& \left.+\left[\sin \left(\phi_{j}\right) \sin \left(\phi_{j+1}\right)+\cos \left(\phi_{j}\right) \cos \left(\phi_{j+1}\right)-1\right]^{2}\right\},
\end{aligned}
$$

which, by elementary trigonometric relations, can be simplified to

$\operatorname{tr}\left(\Delta \mathbf{Q}_{j+1}^{T} \Delta \mathbf{Q}_{j+1}\right)=4\left[1-\cos \left(\phi_{j+1}-\phi_{j}\right)\right]$.

The expansions of $\chi$ and $\phi$ up to first order with respect to $h$ are now considered

$$
\begin{aligned}
& \chi\left(s_{j+1}\right)=\chi\left(s_{j}\right)+\ell \chi^{\prime}\left(s_{j}\right)+o(\ell) \\
& \phi\left(s_{j+1}\right)=\phi\left(s_{j}\right)+\ell \phi^{\prime}\left(s_{j}\right)+o(\ell) .
\end{aligned}
$$

Plugging Eq. (13) and, subsequently, the asymptotic expansions (15) in the energies (8)-(10), following elementary algebraic manipulations, the deformation energy of the discrete system reads as

$$
\begin{aligned}
E & =\sum_{j} E_{a}+E_{b}+E_{c}=\sum_{j}\left\{\frac{\ell^{2} a}{2}\left\|\chi^{\prime}-\frac{\chi^{\prime}}{\left\|\chi^{\prime}\right\|}+o(\ell)\right\|_{s=s_{j}}^{2}\right. \\
& \left.+\ell^{2} b\left[\phi^{\prime 2}+o\left(\ell^{0}\right)\right]_{s=s_{j}}+\frac{\ell^{2} c}{2}\left\|\mathbf{e}(\phi)-\frac{\chi^{\prime}}{\left\|\chi^{\prime}\right\|}+o(\ell)\right\|_{s=s_{j}}^{2}\right\} .
\end{aligned}
$$

The parameters $a, b, c>0$ are now defined to be constants which are independent of $\ell$. These constants are assumed to be related to the stiffnesses of the discrete system by the following scaling power laws

$a=\underline{a} \ell^{-1}, \quad b=\underline{b} \ell^{-1}, \quad c=\underline{c} \ell^{-1}$. 


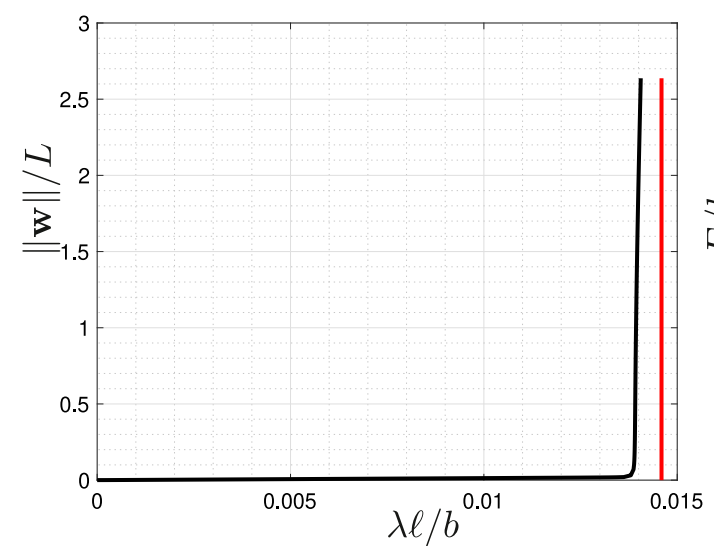

(a)

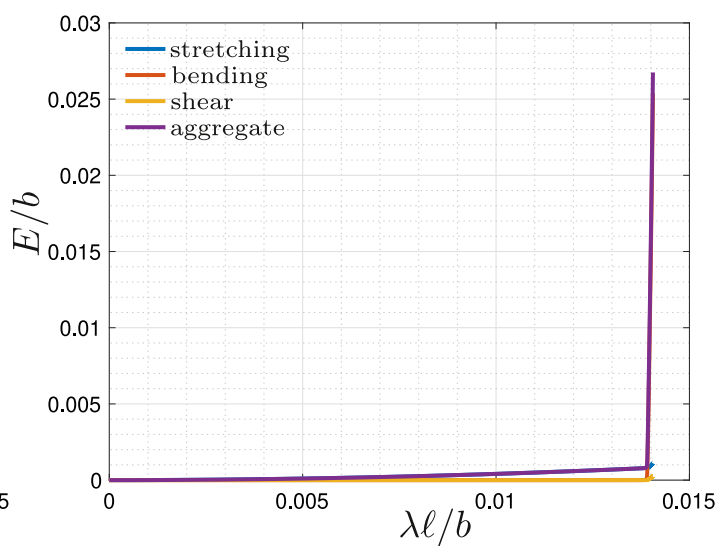

(b)

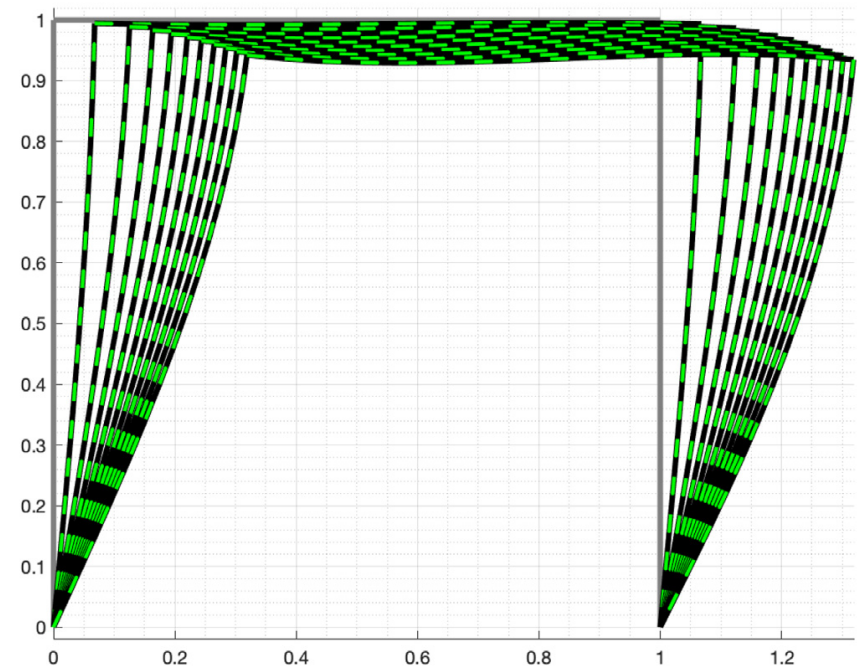

(c)

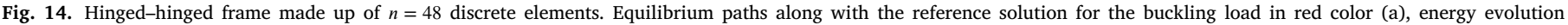
(b), and deformed configuration evolution (c) for $a L^{2} / b=1000$ and $c L^{2} / b=1000(\varepsilon=0.0001)$.

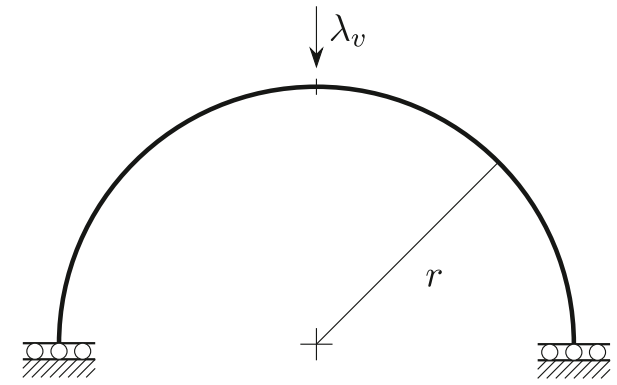

Fig. 15. Semicircular arch on sliders subjected to a vertical load on the midpoint.

Reminding that $\sum_{i} o\left(\ell^{n}\right)=o\left(\ell^{n-1}\right)$, after the application of the scaling laws (17), the global remainder in the energy (16) becomes $o\left(\ell^{0}\right)$. This remainder specifies the deformation energy error between the discrete and the continuum model. The continuum limit can now be obtained by letting $\ell \rightarrow 0$ and reminding that Riemann sums turn into integrals according to $\sum_{j} g\left(s_{j}\right) h \stackrel{\ell \rightarrow 0}{\longrightarrow} \int_{0}^{L} g \mathrm{~d} s$, where $g$ is a real valued function defined on $[0, L]$. Using (16) together with the scaling law (17), the deformation energy for the homogenized macro-model becomes

$$
\tilde{E}=\int_{0}^{L}\left(\frac{a}{2}\left\|\chi^{\prime}-\frac{\chi^{\prime}}{\left\|\chi^{\prime}\right\|}\right\|^{2}+\underline{b} \phi^{\prime 2}+\frac{c}{2}\left\|\mathbf{e}(\phi)-\frac{\chi^{\prime}}{\left\|\chi^{\prime}\right\|}\right\|^{2}\right) \mathrm{d} s .
$$

Let the displacement function $\mathbf{u}:[0, L] \rightarrow \mathbb{R}^{2}$ be defined such that $\chi(s)=s \mathbf{D}_{1}+\mathbf{u}(s)$. Then, the relation $\chi^{\prime}=\mathbf{D}_{1}+\mathbf{u}^{\prime}(s)$ is obtained by deriving with respect to the spatial variable. The following strain measures, which depend non-linearly upon $\chi^{\prime}$ and $\phi$ are clearly identifiable at continuum scale in Eq. (18)

$$
\begin{array}{ll}
\text { 1. }\left\|\chi^{\prime}-\frac{\chi^{\prime}}{\left\|\chi^{\prime}\right\|}\right\| & \text { (extension), } \\
\text { 2. } \phi^{\prime} & \text { (bending), } \\
\text { 3. }\left\|\mathbf{e}(\phi)-\frac{\chi^{\prime}}{\left\|\chi^{\prime}\right\|}\right\| & \text { (shearing). }
\end{array}
$$

Remark that the following representation formula holds for $\mathbf{e}(\phi)$

$\mathbf{e}(\phi)=\cos (\phi) \mathbf{D}_{1}+\sin (\phi) \mathbf{D}_{2}$.

By making use of McLaurin expansions it is easily seen that

$\left\|\chi^{\prime}-\frac{\chi^{\prime}}{\left\|\chi^{\prime}\right\|}\right\|=\left|\mathbf{u}^{\prime} \cdot \mathbf{D}_{1}\right|+o\left(\mathbf{u}^{\prime}\right)$ 


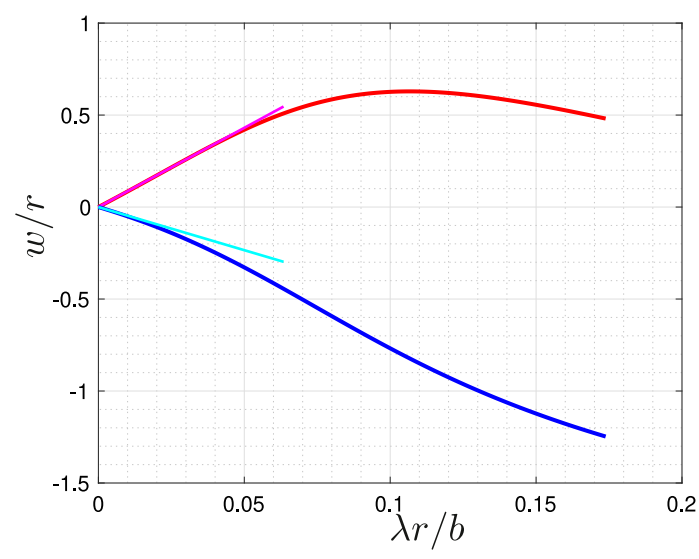

(a)

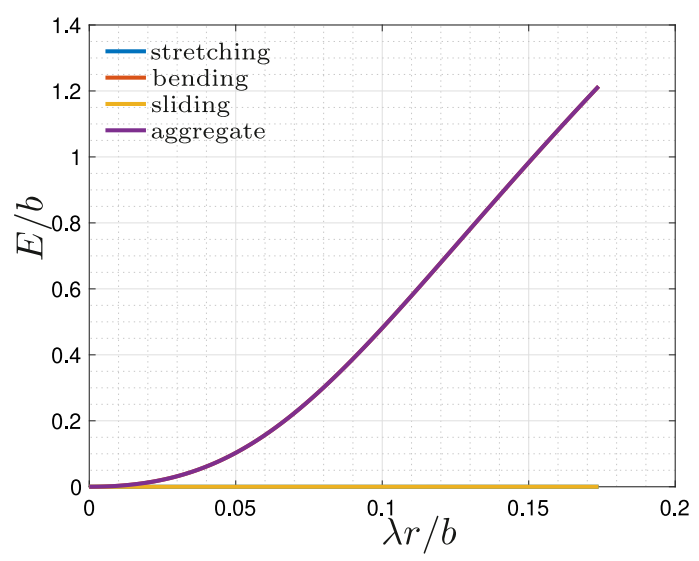

(b)

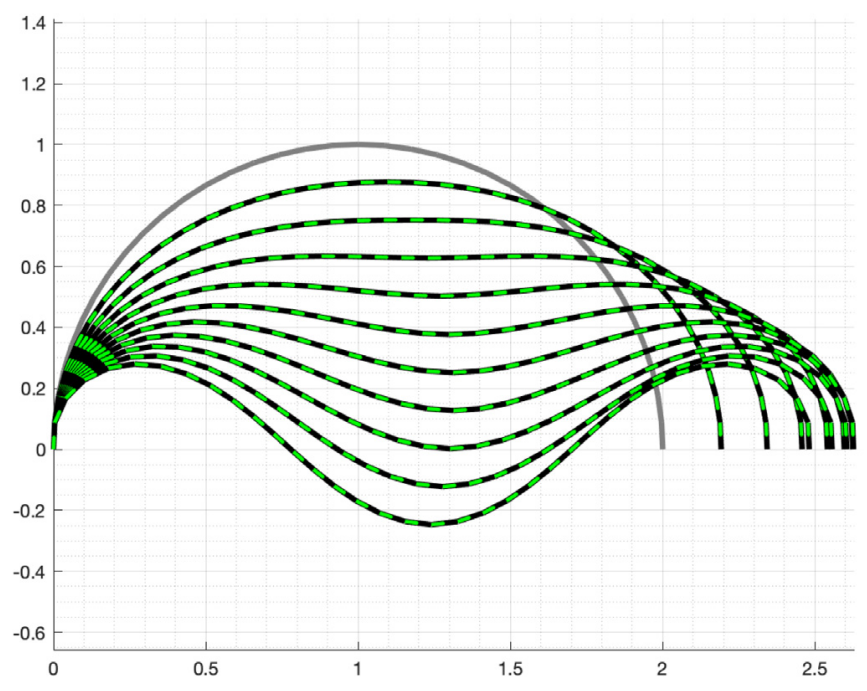

(c)

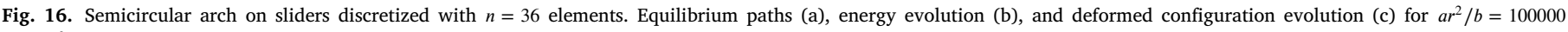
and $c r^{2} / b=100000$.

and that

$\left\|\mathbf{e}(\phi)-\frac{\chi^{\prime}}{\left\|\chi^{\prime}\right\|}\right\|=\left|\phi-\mathbf{u}^{\prime} \cdot \mathbf{D}_{2}\right|+o\left(\mathbf{u}^{\prime}\right)+o(\phi)$.

When small $\mathbf{u}^{\prime}, \phi$ and $\phi^{\prime}$ are considered, then the remainders in Eqs. (21) and (22) can be neglected. In that case, the deformation energy (18) thus recasts as

$\tilde{E}=\int_{0}^{L}\left(\frac{a}{2}\left(\mathbf{u}^{\prime} \cdot \mathbf{D}_{1}\right)^{2}+\underline{b} \phi^{\prime 2}+\frac{c}{2}\left(\phi-\mathbf{u}^{\prime} \cdot \mathbf{D}_{2}\right)^{2}\right) \mathrm{d} s$,

i.e. as in Timoshenko beam model.

\section{Computation of the equilibrium path}

Let us denote the nodal displacement row vector of node $j$ with $\mathbf{u}_{j}$. Let us also denote with $\varphi_{j}$ the rotation angle in Eq. (5), having replaced $\mathbf{Q}$ by $\mathbf{Q}_{j}$. Let us denote the column vector of the independent

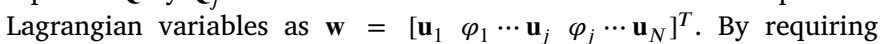
the potential energy to be stationary with respect to the independent Lagrangian variables $\mathbf{w}$ one gets the following nonlinear system of equilibrium equations

$\mathbf{s}[\mathbf{w}]-\mathbf{p}[\lambda]=\mathbf{0}$,

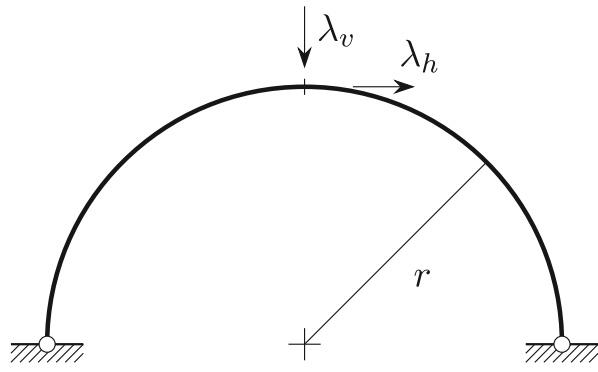

Fig. 17. Hinged semicircular arch subjected to a force on its midpoint.

where the vector $\mathbf{s}$ is the so-called structural reaction, which depends on independent Lagrangian variables $\mathbf{w}$, and $\mathbf{p}[\lambda]$ is the vector of external loads which is parametrized by the dimensionless quantity $\lambda$. The structural reaction $\mathbf{s}$ can be computed as

$\mathbf{s}=\frac{d E}{d \mathbf{w}}$, 


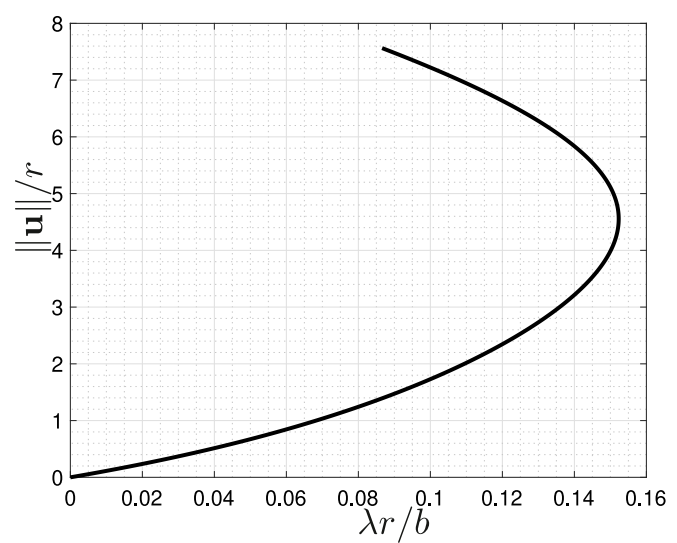

(a)

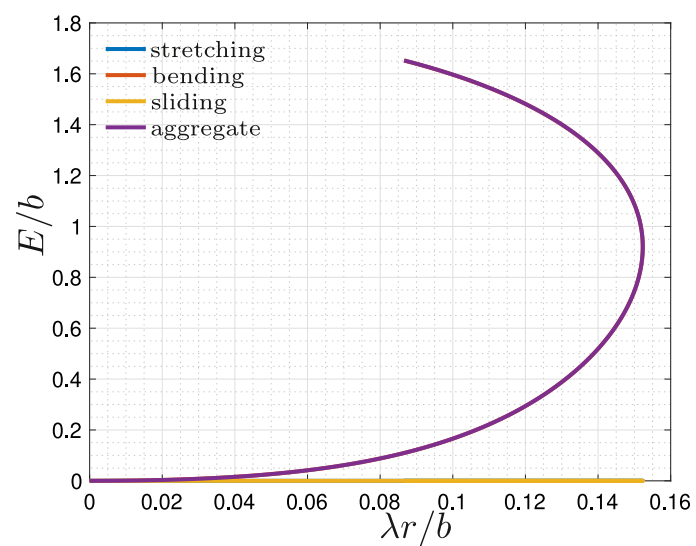

(b)

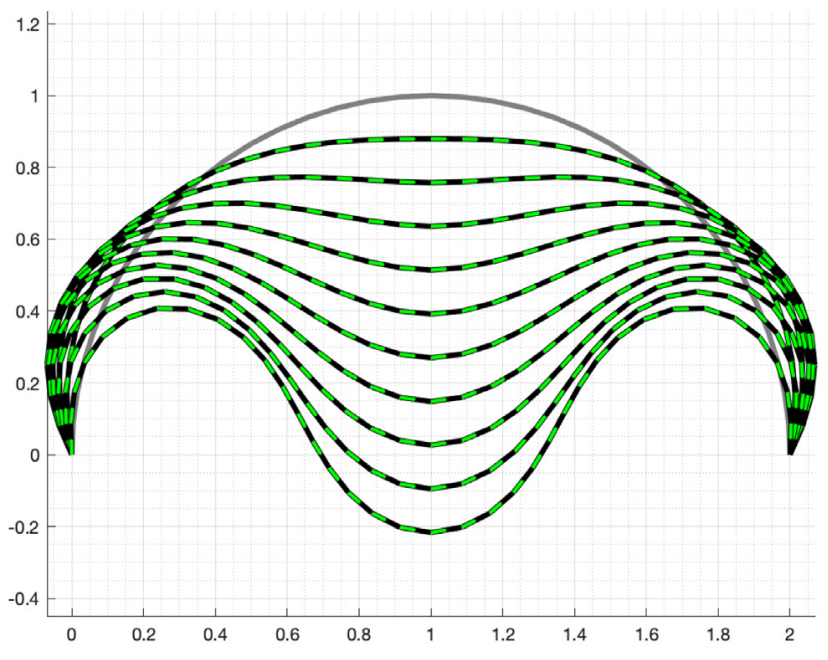

(c)

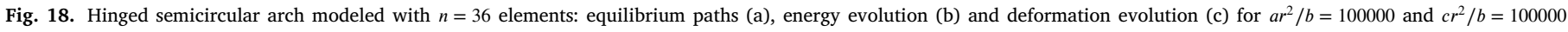
$\left(\lambda_{h}=0\right)$.

where the quantity $E$ denotes the total strain energy of the system obtained by summing up all the contributions (8), (9) and (10) in the system.

The external load vector is assumed have the following linear dependence upon the load parameter $\lambda$

$\mathbf{p}[\lambda]=\mathbf{p}_{0}+\lambda \widehat{\mathbf{p}}$.

It is worth to remark that such an external load representation is convenient when modeling so-called load imperfections or perturbations.

In principle, a stepwise procedure making use of the Newton's method might be exploited to solve the non-linear system of equilibrium equations (24) in the variables $\mathbf{w}$ and $\lambda$. Let the pair $\left(\mathbf{w}_{i}, \lambda_{i}\right)$ be the $i$ th equilibrium point. Assuming that the equilibrium path containing $\left(\mathbf{w}_{i}, \lambda_{i}\right)$ is differentiable, we can obtain an additional, close, equilibrium point $\left(\mathbf{w}_{i}+\Delta \mathbf{w} \lambda_{i}+\Delta \lambda\right)$ by linearizing Eq. (24) in a neighborhood of the $i$ th equilibrium point

$\mathbf{s}\left[\mathbf{w}_{i}\right]+\mathbf{K} \Delta \mathbf{w}-\left(\mathbf{p}_{0}+\left(\lambda_{i}+\Delta \lambda\right) \widehat{\mathbf{p}}\right) \approx \mathbf{0}$,

where the stiffness matrix $\mathbf{K}$ is computed in $\mathbf{w}_{i}$ as

$\mathbf{K}=\frac{d \mathbf{s}}{d \mathbf{W}}$.
In Newton's method, the value of $\Delta \lambda$ is fixed and, noting that $\mathbf{s}\left[\mathbf{w}_{i}\right]-$ $\mathbf{p}\left[\lambda_{i}\right]=0$, (27) gives

$\Delta \mathbf{w}=-\Delta \lambda \mathbf{K}^{-1} \hat{\mathbf{p}}$.

As it is well-known, owing to the necessity of computing the inverse of the stiffness matrix, Newton's method does not converge when $\mathbf{K}$ is zero-determinant or close to it. In order to bypass this limitation, Riks [34] proposed to parametrize the equilibrium path by using its arc-length instead of the dimensionless load parameter $\lambda$ that is indeed fixed at each step in Newton's method. Riks' approach is not affected by the above-mentioned convergence problems, but an additional equation is clearly needed to find the unknown quantity. Riks' arc-length scheme applies a correction to the extrapolation obtained from Newton's formula (29). Let $(\Delta \mathbf{w}, \Delta \lambda)$ be the Newton's extrapolation obtained at the equilibrium point $\left(\Delta \mathbf{w}_{i}, \Delta \lambda_{i}\right)$, then the Riks' correction $(\dot{\mathbf{w}}, \dot{\lambda})$ can be evaluated by making use of the linearization (24) centered in $\left(\mathbf{w}_{i}+\right.$ $\left.\Delta \mathbf{w}, \lambda_{i}+\Delta \lambda\right)$

$\mathbf{s}\left[\mathbf{w}_{i}+\Delta \mathbf{w}\right]+\mathbf{K} \dot{\mathbf{w}}-\left(\mathbf{p}_{0}+\left(\lambda_{i}+\Delta \lambda+\dot{\lambda}\right) \hat{\mathbf{p}}\right) \approx \mathbf{0}$,

where the stiffness matrix $\mathbf{K}$ is now computed in $\mathbf{w}_{i}+\Delta \mathbf{w}$. From (30) follows that $\dot{\mathbf{w}}$ can be computed from

$\dot{\mathbf{w}}=-\mathbf{K}^{-1}\left(\mathbf{s}\left[\mathbf{w}_{i}+\Delta \mathbf{w}\right]-\left(\mathbf{p}_{0}+\left(\lambda_{i}+\Delta \lambda+\dot{\lambda}\right) \hat{\mathbf{p}}\right)\right)$, 


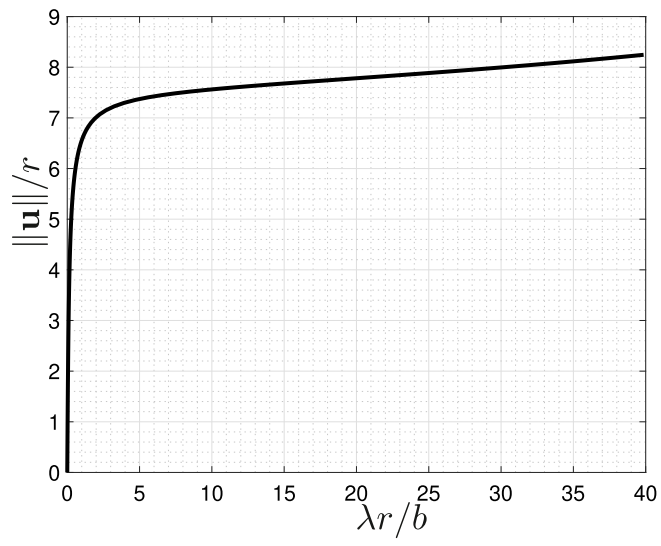

(a)

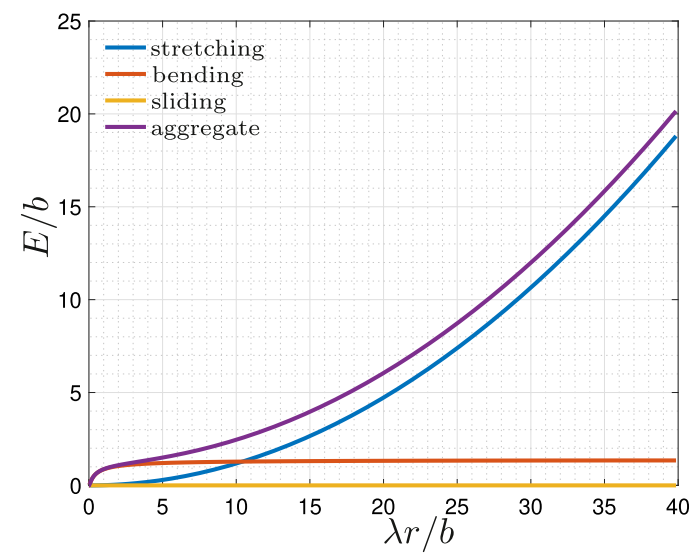

(b)

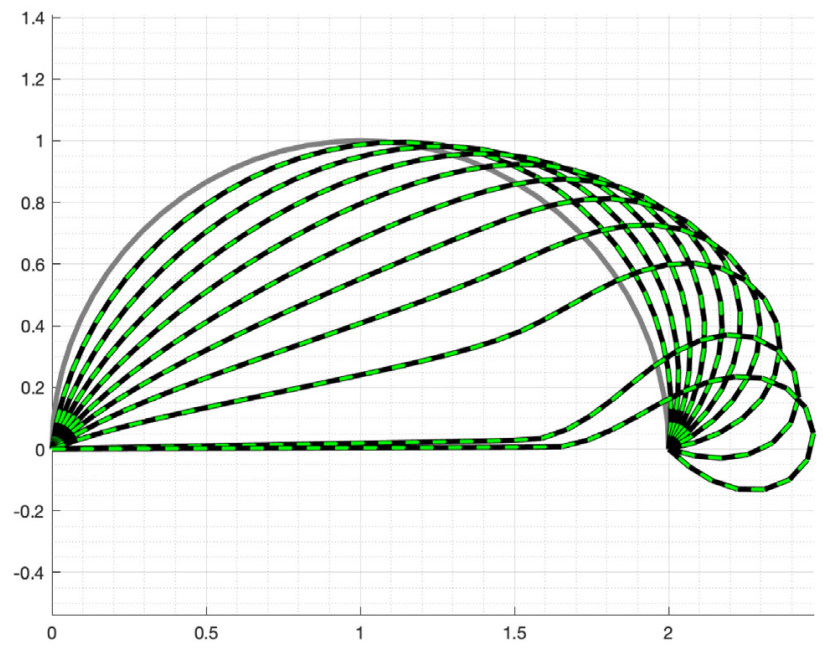

(c)

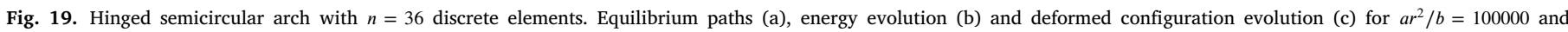
$c r^{2} / b=100000\left(\lambda_{v}=0\right)$.

There are several ways to provide the additional equation required to compensate the appearance of the unknown $\dot{\lambda}$. One of the simplest and most computationally convenient choices is

$$
\Delta \mathbf{w} \cdot \mathbf{K} \dot{\mathbf{w}}=0,
$$

which requires the K-orthogonality between the Newton extrapolation $\Delta \mathbf{w}$ and the Riks' correction $\dot{\mathbf{w}}$. Plugging (31) in (32), by taking into account (29), simple algebra operations lead to the following expression

$\dot{\lambda}=\frac{\widehat{\mathbf{w}} \cdot \mathbf{r}}{\widehat{\mathbf{w}} \cdot \widehat{\mathbf{p}}}$,

where $\mathbf{r}=\mathbf{s}\left[\mathbf{w}_{i}+\Delta \mathbf{w}\right]-\left(\mathbf{p}_{0}+\left(\lambda_{i}+\Delta \lambda\right) \hat{\mathbf{p}}\right)$ denotes the equilibrium equations residual and $\widehat{\mathbf{w}}=\mathbf{K}^{-1} \widehat{\mathbf{p}}$ (cf (29)). It is concluded that, by (31), the Riks' correction $\dot{\mathbf{w}}$ can be computed using the Newton extrapolation $\Delta \mathbf{w}$. Eqs. (33), (31) and (29) completely define the Riks-based algorithm. Riks' correction is applied recursively to the quantities

$\Delta \lambda=\delta\left(\lambda_{i}-\lambda_{i-1}\right)$,

$\Delta \mathbf{w}=\delta\left(\mathbf{w}_{i}-\mathbf{w}_{i-1}\right)$,

until the norm of the equilibrium equations residual is below a given tolerance. The quantity $\delta$ in Eq. (34) is an adaptive coefficient that can be computed as suggested in $[37,38]$

$\delta=1-\frac{r_{l}-n_{l}}{r_{l}+n_{l}}$,

where the quantity $r_{l}$ is the number of Riks' corrections required for converging to the previous equilibrium point and the quantity $n_{l}$ is the expected number of Riks' corrections needed to get convergence. The usual choice for the quantity $n_{l}$ is $n_{l}=5$. At the very first step of the analysis it is assumed $\delta=1$.

\section{A short digression on the shear stiffness coefficient based on classical de Saint-Venant theory}

Among the panoply of situations where the utilization of sheardeformable beam elements is neatly necessary, in this section we shall provide a simple example thereof by means of standard results in the so-called de Saint-Venant theory. Differences between Euler-Bernoulli and Timoshenko beam models can be discussed by considering the cantilever problem for a beam with length $L$ subjected to a transversal force $F$ acting on its free end. It is classically well-known that for the Euler-Bernoulli model the displacement at the free end is equal to $F L^{3} / 3 Y I$, with $Y$ being the Young modulus of the material constituting 


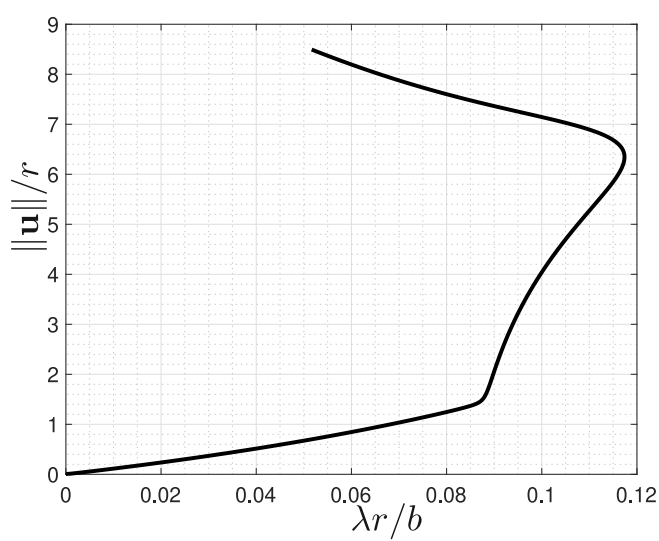

(a)

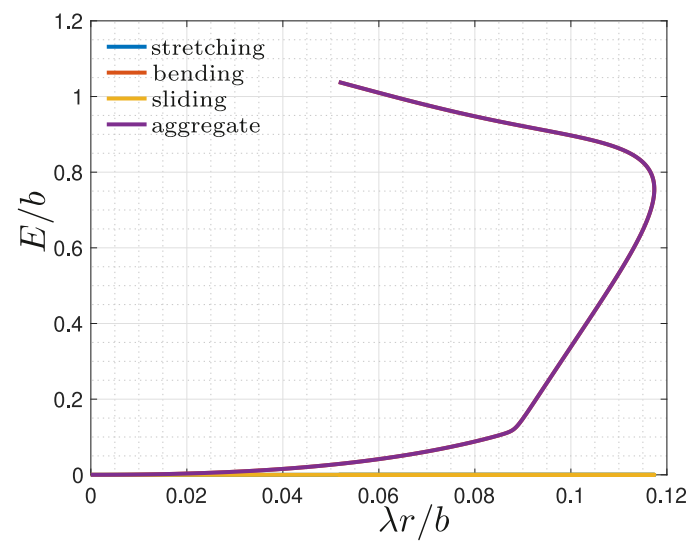

(b)

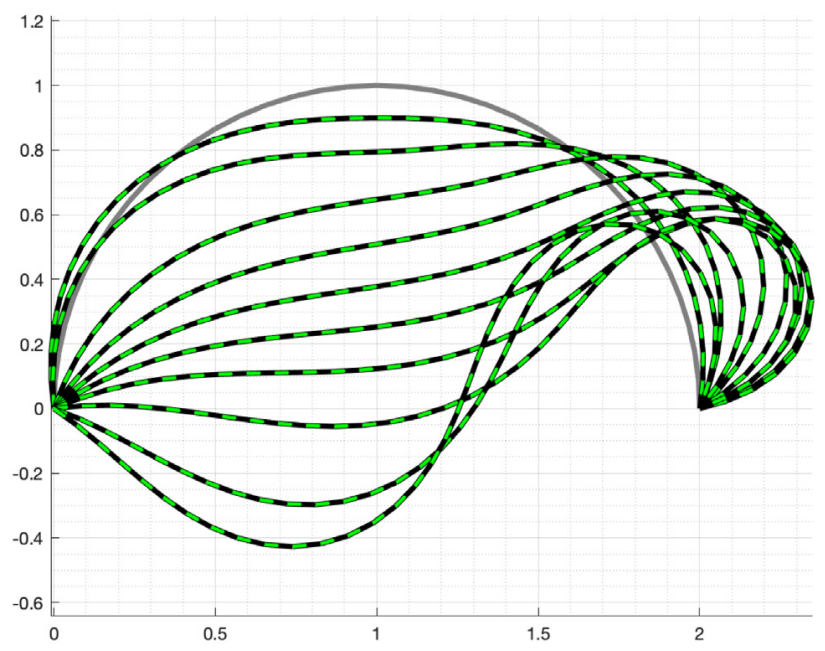

(c)

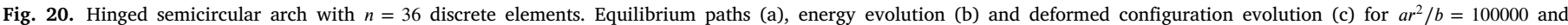
$c r^{2} / b=100000$

the beam and $I$ the moment of inertia of its cross-section. It is also wellknown that in the Timoshenko beam model one must take into account an additional addend due to the shear deformability, i.e., $F L / G A^{*}$, with $G$ being the shear modulus of the material constituting beam and $A^{*}$ the reduced "effective" area of its cross-section. Starting from these two simple results it is concluded that the ratio between the displacement at the free end due to shear, $w_{s}$, and that due to bending, $w_{b}$ can be expressed as

$\frac{w_{s}}{w_{b}}=\frac{\frac{L}{G A^{*}}}{\frac{L^{3}}{3 Y I}}$.

It is therefore clear that this ratio is inversely proportional to the square of the beam length $L$. For instance, if we consider a beam with a rectangular cross-section having a depth $B$ and a height $h$, the ratio in (37) becomes

$\frac{w_{s}}{w_{b}} \propto\left(\frac{h}{L}\right)^{2}$,

where its dependence from the Poisson's ratio of the material constituting the beam has been omitted [39]. To have a rough idea, applying

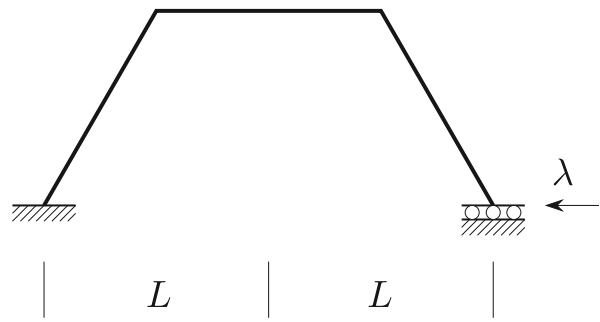

Fig. 21. Half regular hexagon cell.

the previous result to a beam with $\frac{h}{L}=\frac{1}{2}$ (clearly falling out of De Saint-Venant theory hypotheses) then $w_{s}=\frac{1}{4} w_{b}$ and, therefore, the ratio (37) becomes by no means negligible.

At this point, aimed at providing an insight into the possible different contributions which can be given by each cell to behavior of the whole discrete system, a numerical study is presented where three relevant deformation modes (see Fig. 2) are superimposed to the discrete Timoshenko beam system introduced above when only one/ two straight links are considered: 


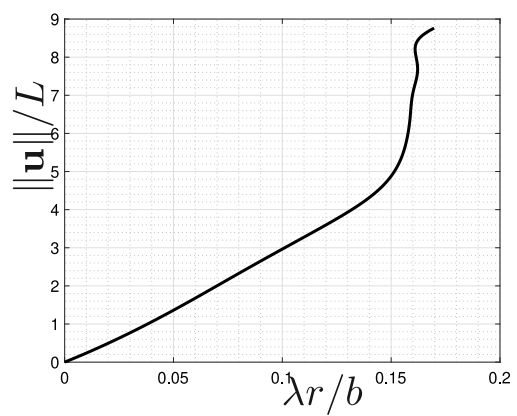

(a)

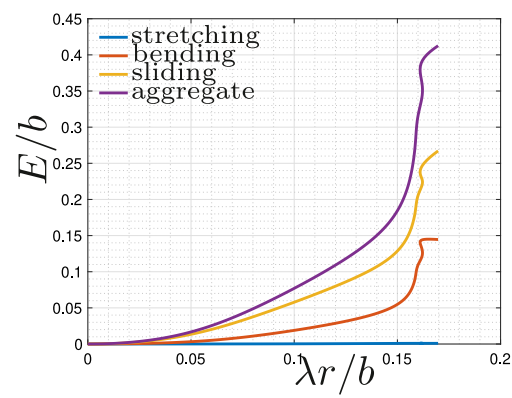

(d)

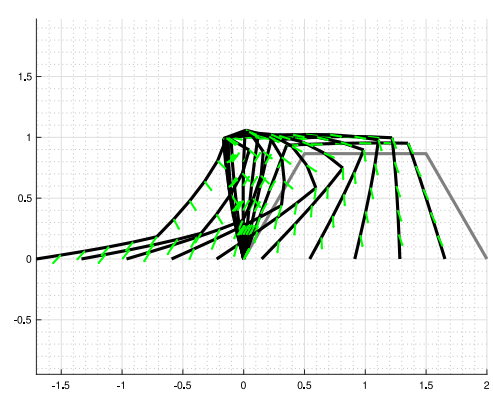

(g)

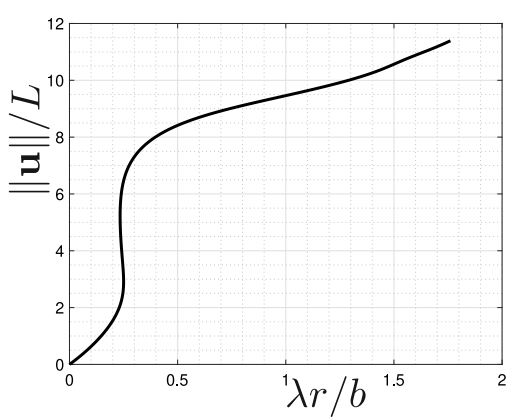

(b)

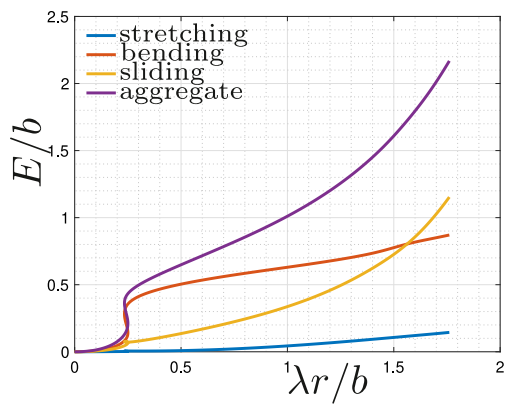

(e)

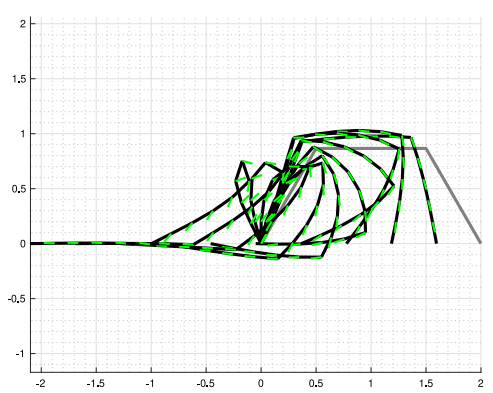

(h)

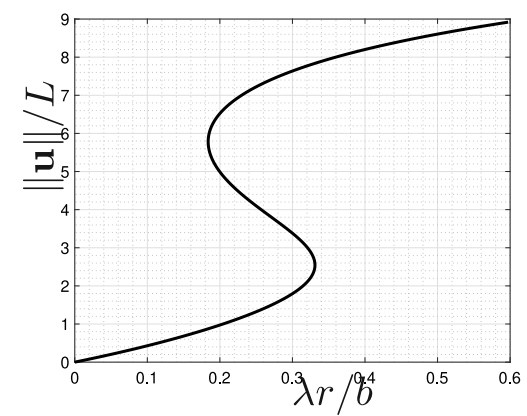

(c)

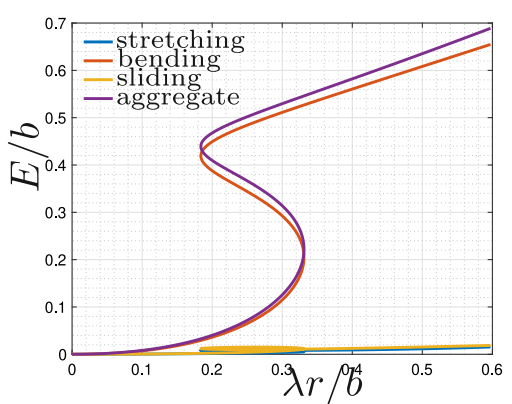

(f)

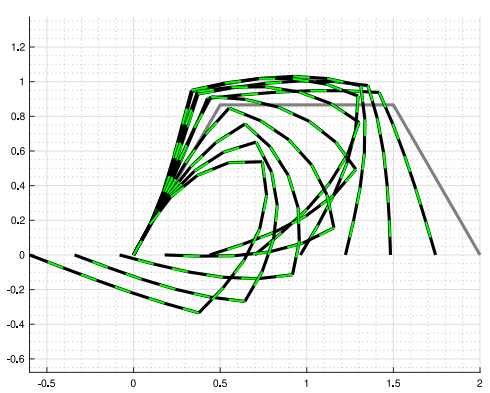

(i)

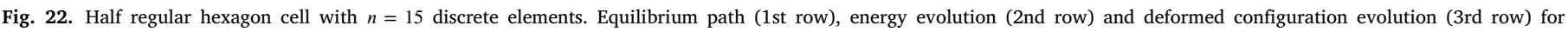
$a L^{2} / b=100$ and $c L^{2} / b=1$ (1st column), $c L^{2} / b=10$ (2nd column) and $c L^{2} / b=100$ (3rd column).

(1) the points $P_{j}$ and $P_{j+1}$ are considered; the condition $p_{j+1}=$ $P_{j+1}+u \mathbf{D}_{1, j}$ is applied while keeping $p_{j}=P_{j}$ and $\mathbf{d}_{1, j}=\mathbf{D}_{1, j}$;

(2) the points $P_{j}$ and $P_{j+1}$ are again considered; the condition $p_{j+1}=$ $P_{j+1}+v \mathbf{D}_{2, j}$ is applied while keeping $p_{j}=P_{j}$ and $\mathbf{d}_{1, j}=\mathbf{D}_{1, j}$;

(3) the points $P_{j}, P_{j+1}$ and $P_{j+2}$ are considered; a rotation of the $\mathbf{d}_{1, j+1}$ is applied while keeping $\mathbf{d}_{1, j}=\mathbf{D}_{1, j}$.

The dimensionless strain energy contributions $E_{a} / a \ell^{2}, E_{b} / b$, and $E_{c} / c \ell^{2}$ have been computed for each test, with $\ell$ being the link length. Fig. 3 shows the strain energy as divided into the above mentioned scaled energy contributions ( $a$ - stretch; $b$ - bending; $c$ - shear) as functions of the scaled parameters $u, v$ and $\varphi$, being the latter the superimposed angle in-between the vectors $\mathbf{d}_{1, j+1}$ and $\mathbf{d}_{1, j}$. Remark that the first test entails only extension deformation, the second test entails stretch and shear deformations and, finally, the third test entails stretch, shear, and bending deformations. Fig. 4 shows the shear-tostretch strain energy ratio versus the dimensionless displacement $v / \ell$ for the second test as the ratio $c / a$ from 0.1 to 10 varies and for a constant $b$. Fig. 5 shows the dimensionless shear strain energy $E_{c} / c \ell^{2}$ versus $\varphi$ for the third test as the non-dimensional stiffness ratio $c \ell^{2} / b$ varies from 0.1 to 10 and for a constant $a$. We remark that in this case the shear-to-bending strain energy ratio $E_{c} / E_{b}$ is constant as $\varphi$ increases until $\pi$.
These last two plots highlight well the influence of the shear deformability. It is remarkable that, owing to the chosen material behaviors (i.e. Hookean) and to the (geometric) strains definition, the shear energy contribution becomes relatively less important when the control variable increases, i.e. for large deformations.

\section{Buckling of frames and arches}

In this section, aimed at testing the discrete Timoshenko beam formulation presented above, several problems inspired by classic tests related to buckling of frames and arches are presented and solved by the Riks' strategy introduced above. The main goal of this analysis is to show the peculiarities of the obtained mechanical behavior when the stiffness parameters change. The analysis will be especially focused on understanding said mechanical behavior when the shear stiffness changes leading from Euler-Bernoulli, i.e. $c \rightarrow \infty$, to Timoshenko model.

The following case studies will be considered: (i) Roorda's frame; (ii) hinged-hinged frame; (iii) hinged semicircular arch and (iv) halfhexagonal cell. Moreover, the static equilibrium position problem for a cantilever beam subjected to a transversal force on its free tip will be considered. For this last problem, besides the trivial final configuration also an exotic equilibrium configuration will be computed in large 


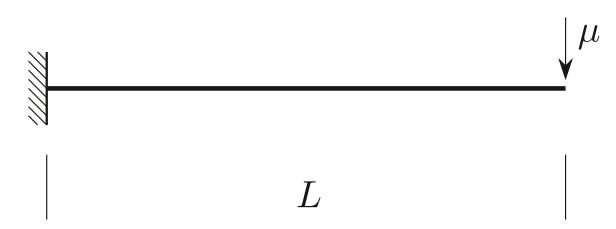

(a)

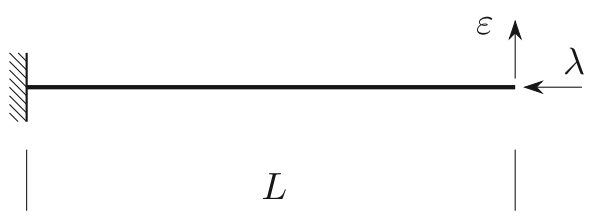

(b)

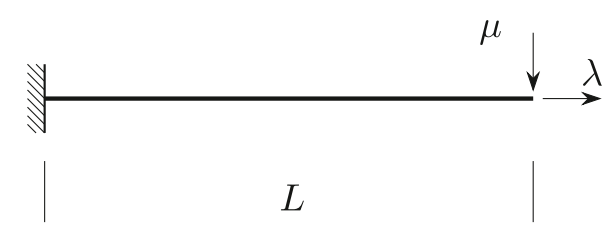

(c)

Fig. 23. Cantilever under a shear load $\mu$ (a), compression $\lambda$ and shear imperfection load $\varepsilon$ (b), shear $\mu$ and traction load $\lambda$ (c).

deformations, finding agreement with previous mathematical and numerical investigations [40]. In addition, thanks to the stepwise strategy which we used, we are able to trace the complete path to achieve such a curled configuration.

All the problems mentioned above require to fix the constitutive parameters of the Hencky beam, i.e. the stiffness parameters $a, b$ and c. Almost always, reference solutions are given for inextensible beams and neglecting shear deformability, therefore we start by considering the identification of the bending stiffness parameter $b$. A simple way to estimate $b$ consists in considering a cantilever beam with length $L$ subjected to a couple $M$ acting on its free end. It is well-known that the rotation of the free end $\varphi$ is equal to $M L / E I$. On the other hand, for a beam discretized with $n$ links a measure of the rotation on the free end is $(n-1) M / b$ where $b$ denotes the bending stiffness parameter corresponding to the chosen bending strain measure. ${ }^{2}$ Matching the free end rotation of the continuum and of the discrete model it is possible to estimate the stiffness $b$. Alternatively, one can choose $b$ so as to match the buckling load of the continuum cantilever with that of the discrete one for a sufficiently large number $n$ of links, obtaining practically the same result. The same procedures can be used with proper adaptations to estimate the stiffnesses $a$ and $c$.

\subsection{Roorda's frame}

The Roorda's frame problem, see [41], is depicted in Fig. 6. The frame is loaded with a vertical force $\lambda$ on the corner and a horizontal load $\varepsilon$, representing an imperfection load, on the midpoint of the vertical beam.

The buckling load $\lambda_{\text {ref }}$ for this frame is reported in [41] by neglecting both the axial and shear deformability.

Fig. 7 shows equilibrium paths obtained by increasing the number of discrete elements $n$ used to model the beams of the frame. All element lengths are equal to $\ell$. Each point of the equilibrium path

\footnotetext{
2 We remark that the bending strain measure used for the proposed model
} is given by Eq. (9). is a dimensionless pair formed by the dimensionless load $\lambda \ell / b$ and the corresponding dimensionless displacement $\|\mathbf{w}\| / L$, where $\mathbf{w}$ denotes the vector collecting the Lagrangian parameters used to model the displacements of the frame.

Fig. 8 shows the subsequent equilibrium configurations obtained by using 32 elements of equal length, dimensionless stiffnesses $a L^{2} / b=$ 100 and $c L^{2} / b=1$, and an imperfection load with magnitude $\varepsilon=0.001$. Henceforth, black is used to graphically present displacements, whereas green arrows indicate rotations. For the same data, Fig. 9 shows the dimensionless strain energy $E / b$ corresponding to the dimensionless load parameter $\lambda L / b$. Aimed at highlighting the prevalent contributions to the dimensionless strain energy, strain energy is split into stretch, bending and shear parts. Fig. 10 shows the influence of the magnitude $\varepsilon$ of the imperfection load on the dimensionless equilibrium path $(\lambda \ell / b$, $\|\mathbf{u}\| / L$ ) obtained by considering the dimensionless stiffnesses $a L^{2} / b=$ 1000 and $c L^{2} / b=1000$. Fig. 11 shows the influence of the dimensionless parameter $a L^{2} / b$. By using 32 elements, $\varepsilon=0.001$ and $c L^{2} / b=1$, the equilibrium path, the strain energy evolution and the deformed configuration evolution are plotted for $a L^{2} / b=1$ (left) and $a L^{2} / b=$ 10000 (right). Finally, Fig. 11 shows the influence of the dimensionless parameter $c L^{2} / b$. By using 32 discrete elements, $\varepsilon=0.0001$ and $a L^{2} / b=1000$, the equilibrium path, the strain energy evolution and the deformed configuration evolution are plotted for $c L^{2} / b=10$ (left) and $c L^{2} / b=100000$ (right) (see Fig. 12).

\subsection{Hinged-hinged frame}

The hinged-hinged frame depicted in Fig. 13, with depth and height equal to $L$, shall now be addressed. Loads act on the upward corners compressing the vertical beams and, in addition, an imperfection horizontal load with magnitude $\varepsilon$ is considered. Reference solution was given in neglecting, also in this case, the extensibility and the shear deformation of the beams constituting the frame.

Fig. 14 account for a numerical simulation performed by using 48 discrete elements with equal length, an imperfection load magnitude $\varepsilon=0.0001, a L^{2} / b=1000$ and $c L^{2} / b=1000$. Particularly, Fig. 14(a) reports the computed dimensionless equilibrium path (the reference solution, in red color, for the buckling load given in [42] is also reported), Fig. 14(b) reports the evolution of the dimensionless energy as split into stretch, bending and shear parts. Finally, Fig. 14(c) shows the evolution of the deformed configuration for subsequent load steps.

\subsection{Semicircular arch on sliders}

In this subsection a semicircular arch on sliders of radius $r$ is considered, see Fig. 15. The arch is loaded at its midpoint with a vertical force $\lambda_{v}$.

The simulation uses 36 discrete elements with equal length, and dimensionless stiffness parameters $a r^{2} / b=100000$ and $c r^{2} / b=100000$. The bending stiffness $b$ was identified by using the same procedure sketched at the beginning of this section. Fig. 16(a) shows for this set of data the dimensionless equilibrium path for the lengthening of the points bounded with the sliders (in color red) and the vertical displacement of the arch midpoint (in color blue). On the same figure is also drawn the reference solution, in magenta and cyan color, respectively, given in [43]. Fig. 16(b)-(c) report the strain energy evolution - again split in stretch, bending and shear parts - and the deformed configuration evolution, respectively.

\subsection{Hinged semicircular arch}

With the same bending stiffness parameter $b$ used in the previous test we consider the case reported in Fig. 17.

Fig. 18 accounts for the case $\lambda_{h}=0$ while the other stiffnesses are left unchanged (first simulation): dimensionless equilibrium path 


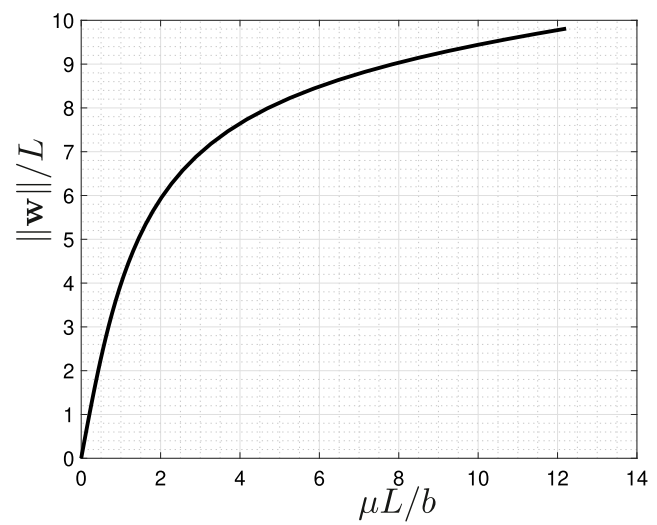

(a)

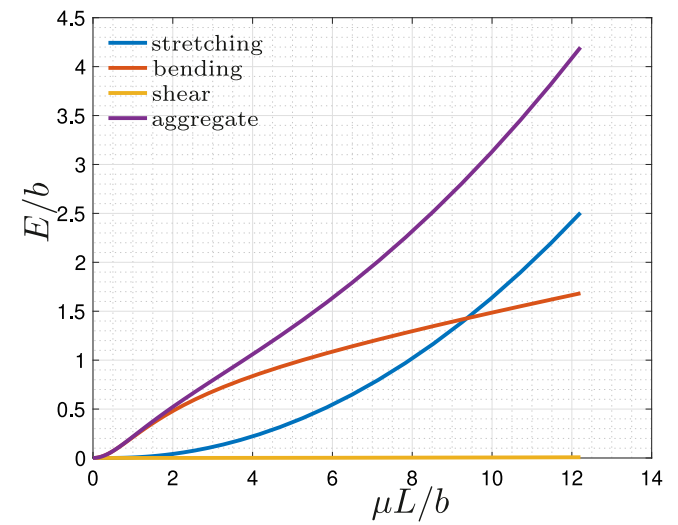

(b)

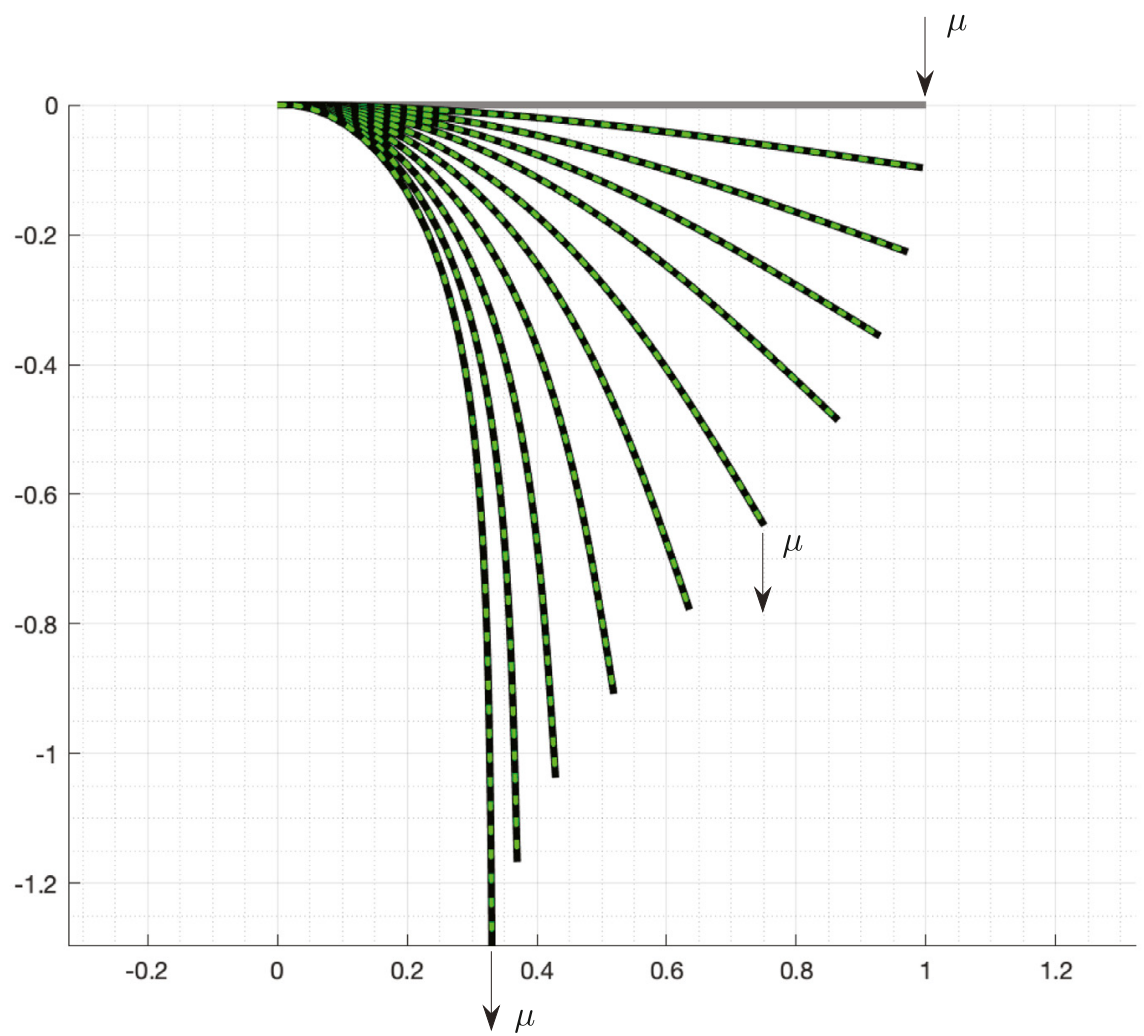

(c)

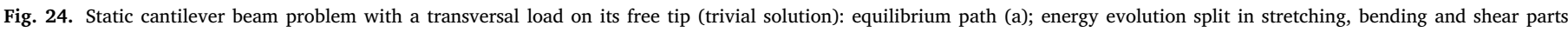
(b); deformation evolution (c).

(a), dimensionless strain energy evolution (b) - again split in stretch, bending and shear parts - and deformed configuration evolution (c) are plotted.

Fig. 19 accounts for the case $\lambda_{v}=0$ while the other stiffnesses are left unchanged (second simulation): dimensionless equilibrium path (a), dimensionless strain energy evolution (b) - again split in stretch, bending and shear parts - and deformed configuration evolution (c) are plotted.

Fig. 20 collects the results of the simulation by considering the case of vertical force $\lambda_{v}$ along with a horizontal force as imperfection $\lambda_{h}=0.01$ while the other stiffnesses are left unchanged (third simulation): dimensionless equilibrium path (a), dimensionless strain energy evolution (b) - again split in stretch, bending and shear parts - and deformed configuration evolution (c) are plotted.

\subsection{Half regular hexagon cell}

This case study is frequently studied in the mechanics of architectured materials. A half regular hexagon is analyzed with the boundary conditions sketched in Fig. 21.

Fig. 22 accounts for simulation performed by using $n=15$ discrete elements and $a L^{2} / b=100$. The equilibrium path, the evolutions of the strain energy and of the deformed configuration are plotted for $c L^{2} / b=1$ (1st column), $c L^{2} / b=10$ (2nd column) and $c L^{2} / b=100$ (3rd column). All the plots show the influence of the shear stiffness 


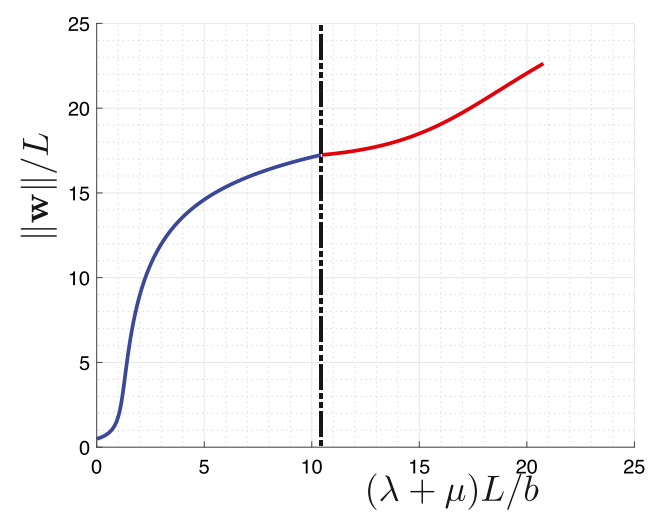

(a)

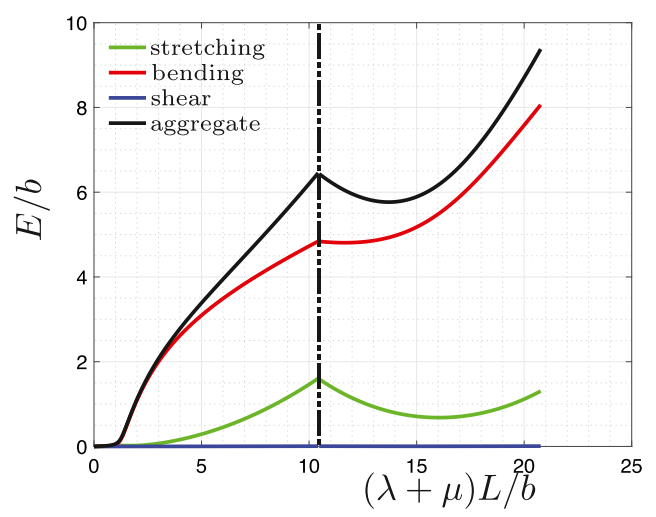

(b)

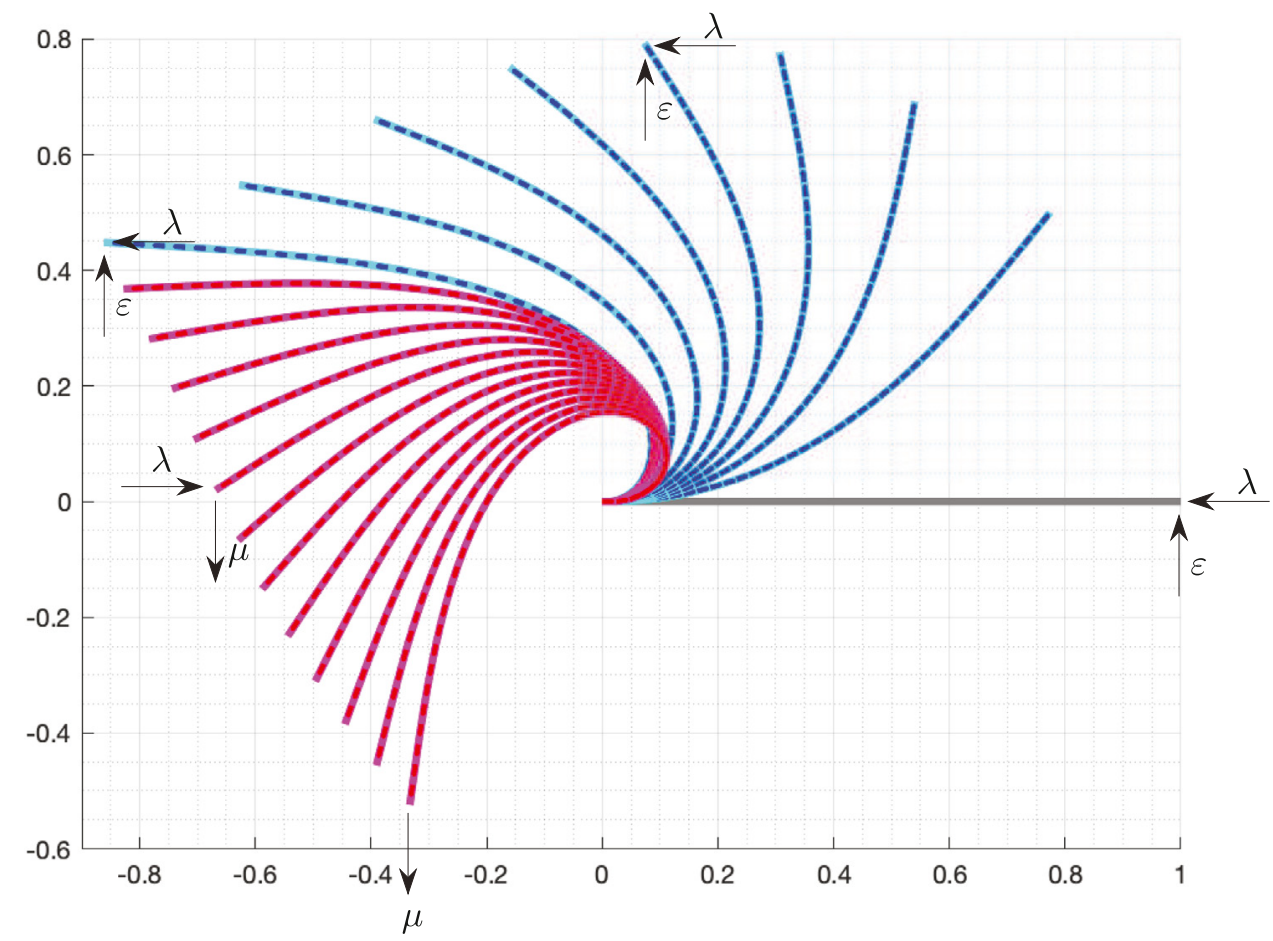

(c)

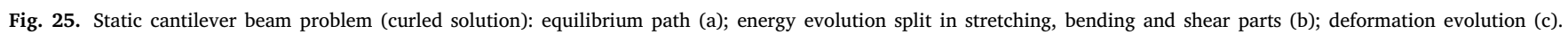

coefficient $c$ both on the equilibrium path and on the deformation evolution.

\subsection{Curled equilibrium configuration for a cantilever beam}

This case study has been chosen aimed at verifying some results presented in $[7,44]$. More precisely, by referring to a cantilever beam with length $L$, we look for equilibrium configurations when a transversal load $\mu$ is applied on the tip, see Fig. 23(a).

By using 40 elements, each one characterized by the nodal displacements of its ends - four Lagrangian parameters - and by the rotation - one Lagrangian parameter - and for the stiffness ratios $a L^{2} / b=100$ and $c L^{2} / b=10000$, an analysis developed by using the tools exposed in the foregoing produces the following results. Fig. 24, where besides the equilibrium path (a) and the energy evolution (b), split in stretching, bending and shear parts, shows also the evolution of the deformation (c), in a stroboscopic fashion, when the load parameter $\mu$ increases.

It is possible to reach different equilibrium configurations for the same transversal tip force. Aimed at finding the curled configuration obtained by said (only) transversal tip force, a loading process has been devised which consists in two consecutive steps. First, the buckling of the cantilever is achieved by increasing with a compression load $\lambda$ on the tip complemented by a fixed transversal imperfection load $\varepsilon$, see Fig. 23(b). Successively, starting from the final equilibrium point obtained by the first loading, a transversal tip force, which rapidly nullifies the imperfection $\varepsilon$, along with a horizontal force, which gradually nullifies the buckling force of the previous step, are applied (see Fig. 23(c)). In this way, we reach the desired equilibrium configuration (see Fig. 25) different from that presented in Fig. 24 with the same final force. 
Fig. 25 reports the numerical simulation carried out by combining the two loading processes, separated by a dot-dashed line, mentioned above. In order to distinguish the two loading processes different colors have been used for the deformations: cold colors - blue and cyan for the first one and warm colors - red and magenta - for the second one, see Fig. 25(a) and (c). As before, Fig. 25(b) shows the total energy evolution as split in stretching, bending and shear parts.

\section{Concluding remarks and future challenges}

In this work we considered the problem of predicting the equilibrium shapes of largely deformed slender bodies in which extension and shear phenomena cannot be neglected. We limited our analysis to the case of planar deformations, albeit the techniques presented here can be easily generalized to the case of bodies moving in 3D space: in fact, Section 2 presents a formulation which is already suitable for $3 \mathrm{D}$ motions.

In the literature Timoshenko beam model is formulated to the same aim. One has, however, to make two remarks concerning Timoshenko model: ( $i$ ) there are different possibilities to generalize Timoshenko theory, which is intrinsically linear, to the case of large deformations and large displacements (see [7,45]); (ii) Timoshenko model is a continuum model: therefore, when it is not possible to find closed form solutions, its discretization is needed. This discretization, usually, is chosen without taking into account the true nature of the mechanical system which is described and the mathematical structure of the deformation energy postulated in Timoshenko models. Therefore many undesired difficulties (like locking) arise: to overcome these difficulties remarkable efforts were required (see e.g. $[46,47]$ ).

In the present paper we formulated a Lagrangian Hencky type nonlinear model aimed to model the mechanical behavior of shareable extensible slender deformable bodies. It is intrinsically discrete and the introduced Lagrangian parameters have an immediate mechanical interpretation in terms of extension, bending and shear deformation. The postulated energy has a clear physical interpretation and can be easily used in the formulation of the numerical code used for computations. Moreover the algorithm which we use for calculating the deformed shapes is very efficient and allows for the determination of equilibrium shapes also in presence of multiple solutions of the same deformation problem.

Our motivation in formulating a more efficient computing tool alternative to Timoshenko theory is related to our intention to consider complex beam structures in novel metamaterials design. The formulated Lagrangian Hencky type non-linear model is clearly inspired by the Hencky's dissertation and it is coded in a general stepwise solution strategy based essentially on an adaptive Riks' arc-length approach which proves to be very efficient for reconstructing equilibrium path containing limit points. Several numerical tests, mostly suggested by the intended applications to the design of architectured materials, have been performed to highlight the peculiarities of the proposed discrete model: indeed the modularity of the formulation in terms of discrete elements is easily adaptable for simulating the non-linear behavior of latticed metamaterials. It is foreseen that, for this reason, such a formulation could be used as a short-cut tool to guide the development, optimization and experimentation of new metamaterials and, possibly, also to validate their continuum description. The influence of shear stiffness on the overall mechanical behavior of considered structures has been investigated too. Such a stiffness - as well as the others - can be purposely tuned to achieve desired behaviors.

Open problems and challenges that could be tackled in the next future include: (i) a careful analysis of the stiffness parameters used to characterize the elastic response of the whole beam in the largedeformation regime, and in general when de Saint-Venant estimate of stiffness parameters does not apply; such parameters should be related to the material constitutive parameters of the material constituting the meso-beams such as the Young and tangential moduli, and to geometrical parameters of the beams cross-section, as the area, the shear correction factor, and the moment of inertia; (ii) the development of functionally graded materials, meaning those materials having stiffness parameters which are varying along the beam axis; an extended campaign of numerical simulations might unveil new and exotic mechanical behaviors, see [48-51]; (iii) the development of continuum models, as those developed and exploited, e.g., in [52-60], aimed at describing for large displacements systems with many discrete elements of the type presented here; besides being useful in unveiling so-called emerging phenomena, continuum models could help in identifying stiffness parameters; (iv) the exploitation of the presented approach to provide a validation and insight into new and existing approaches for the extension of stability theory in classical elastic media to micromorphic, strain-gradient $[61,62]$, and Cosserat media, see, e.g., $[12,63-70]$; $(v)$ the extension of the presented approach to problems where dynamics effects are non-negligible, see, e.g., [71-73], like those studied in the active control of vibrations [74]; (vi) the validation of continuum approaches to the study of plane and curved structures moulded as, e.g., shells and tubes, see [75-77]. It seems also interesting the extension of the present work to kinematically higherorder - Levison-Bickford-Reddy type - beam models allowing curved cross-sectional fibers, see [78].

\section{Declaration of competing interest}

The authors declare that they have no known competing financial interests or personal relationships that could have appeared to influence the work reported in this paper.

\section{Acknowledgment}

Emilio Turco was supported by the University of Sassari, Italy (Fondo di Ateneo per la ricerca 2019). This contribution is gratefully acknowledged.

\section{References}

[1] S.R. Eugster, Geometric Continuum Mechanics and Induced Beam Theories, in: Lecture Notes in Applied and Computational Mechanics, vol. 75, Springer, 2015.

[2] D.N. Arnold, A.L. Madureira, S. Zhang, On the range of applicability of the Reissner-Mindlin and Kirchhoff-Love plate bending models, J. Elasticity 67 (2002) 171-185.

[3] E. Benvenuto, La Scienza Delle Costruzioni Ed Il Suo Sviluppo Storico, first ed., Sansoni, 1981.

[4] R. Ballarini, The da Vinci-Euler-Bernoulli beam theory? Mech. Eng. Mag. Online 07 (2003).

[5] C. Truesdell, The Mechanics of Leonardo Da Vinci, Springer, Berlin, Heidelberg, 1968.

[6] I. Elishakoff, Who developed the so-called Timoshenko beam theory? Math. Mech. Solids (2019) 1-20, http://dx.doi.org/10.1177/1081286519856931.

[7] A. Della Corte, A. Battista, F. dell'Isola, P. Seppecher, Large deformations of Timoshenko and Euler beams under distributed load, Z. Angew. Math. Phys. 70 (2) (2019) 52.

[8] V.L. Berdichevskii, L.A. Starosel'skii, On the theory of curvilinear Timoshenkotype rods, J. Appl. Math. Mech. 47 (6) (1983) 809-817.

[9] A. Cazzani, F. Stochino, E. Turco, On the whole spectrum of Timoshenko beams. Part I: a theoretical revisitation, Z. Angew. Math. Phys. 67 (24) (2016) 1-30.

[10] A. Cazzani, F. Stochino, E. Turco, On the whole spectrum of Timoshenko beams. Part II: further applications, Z. Angew. Math. Phys. 67 (25) (2016) 1-21.

[11] A.-T. Luu, N.-I. Kim, J. Lee, Isogeometric vibration analysis of free-form Timoshenko curved beams, Meccanica (2014).

[12] V. Balobanov, J. Niiranen, Locking-free variational formulations and isogeometric analysis for the Timoshenko beam models of strain gradient and classical elasticity, Comput. Methods Appl. Mech. Engrg. 339 (2018) 137-159.

[13] J. Kiendl, F. Auricchio, T.J.R. Hughes, A. Reali, Single-variable formulations and isogeometric discretizations for shear deformable beams, Comput. Methods Appl. Mech. Engrg. 284 (2015) 988-1004.

[14] A. Cazzani, F. Stochino, E. Turco, An analytical assessment of finite elements and isogeometric analysis of the whole spectrum of Timoshenko beams, J. Appl. Math. Mech./Z. Angew. Math. Mech. 96 (10) (2016) 1220-1244.

[15] L.R. Meza, G.P. Phlipot, C.M. Portela, A. Maggi, L.C. Montemayor, A. Comella, D.M. Kochmann, J.R. Greer, Reexamining the mechanical property space of three-dimensional lattice architectures, Acta Mater. 140 (2017) 424-432. 
[16] L.R. Meza, S. Das, J.R. Greer, Strong, lightweight, and recoverable three-dimensional ceramic nanolattices, Science 345 (6202) (2014) 1322-1326.

[17] A. Gross, P. Pantidis, K. Bertoldi, S. Gerasimidis, Correlation between topology and elastic properties of imperfect truss-lattice materials, J. Mech. Phys. Solids 124 (2019) 577-598.

[18] Z. Vangelatos, K. Komvopoulos, C. Grigoropoulos, Vacancies for controlling the behavior of microstructured three-dimensional mechanical metamaterials, Math. Mech. Solids 24 (2) (2018) 577-598.

[19] Z. Vangelatos, V. Melissinaki, M. Farsari, K. Komvopoulos, C. Grigoropoulos, Intertwined microlattices greatly enhance the performance of mechanical metamaterials, Math. Mech. Solids 24 (8) (2019) 2636-2648.

[20] E. Marino, Isogeometric collocation for three-dimensional geometrically exact shear-deformable beams, Comput. Methods Appl. Mech. Engrg. 307 (2016) 383-410.

[21] E. Marino, Locking-free isogeometric collocation formulation for threedimensional geometrically exact shear-deformable beams with arbitrary initial curvature, Comput. Methods Appl. Mech. Engrg. 324 (2017) 546-572.

[22] J.C. Simo, A finite strain beam formulation. The three-dimensional dynamic problem. Part I, Comput. Methods Appl. Mech. Engrg. 49 (1) (1985) 55-70.

[23] A. Della Corte, F. dell'Isola, R. Esposito, M. Pulvirenti, Equilibria of a clamped Euler beam (Elastica) with distributed load: Large deformations, Math. Models Methods Appl. Sci. 27 (8) (2017) 1391-1421.

[24] F. dell'Isola, P. Seppecher, M. Spagnuolo, E. Barchiesi, F. Hild, T. Lekszycki, I. Giorgio, L. Placidi, U. Andreaus, M. Cuomo, S.R. Eugster, A. Pfaff, K. Hoschke, R. Langkemper, E. Turco, R. Sarikaya, A. Misra, M. De Angelo, F. D'Annibale, A. Bouterf, X. Pinelli, A. Misra, B. Desmorat, M. Pawlikowski, C. Dupuy, D. Scerrato, P. Peyre, M. Laudato, L. Manzari, P. Göransson, C. Hesch, S. Hesch, P. Franciosi, J. Dirrenberger, F. Maurin, Z. Vangelatos, C. Grigoropoulos, V. Melissinaki, M. Farsari, W. Muller, B. Emek Abali, C. Liebold, G. Ganzosch, P. Harrison, R. Drobnicki, L. Igumnov, F. Alzahrani, T. Hayat, Advances in pantographic structures: design, manufacturing, models, experiments and image analyses, Contin. Mech. Thermodyn. 31 (4) (2019) 1231-1282.

[25] F. dell'Isola, P. Seppecher, J.J. Alibert, T. Lekszycki, R. Grygoruk, M. Pawlikowski, D.J. Steigmann, I. Giorgio, U. Andreaus, E. Turco, M. Gołaszewski, N. Rizzi, C. Boutin, V.A. Eremeyev, A. Misra, L. Placidi, E. Barchiesi, L. Greco, M. Cuomo, A. Cazzani, A. Della Corte, A. Battista, D. Scerrato, I. Zurba Eremeeva, Y. Rahali, J.-F. Ganghoffer, W. Muller, G. Ganzosch, M. Spagnuolo, A. Pfaff, K. Barcz, K. Hoschke, J. Neggers, F. Hild, Pantographic metamaterials: an example of mathematically driven design and of its technological challenges, Contin. Mech. Thermodyn. 31 (4) (2019) 851-884

[26] H. Hencky, Über die angenäherte Lösung von Stabilitätsproblemen im Raum mittels der elastischen Gelenkkette (Ph.D. thesis), Engelmann, 1921.

[27] C.M. Wang, H. Zhang, R.P. Gao, W.H. Duan, N. Challamel, Hencky bar-chain model for buckling and vibration of beams with elastic end restraints, Int. J. Struct. Stab. Dyn. (2014) 1540007.

[28] N. Challamel, A. Kocsis, C.M. Wang, Discrete and non-local elastica, Int. J. Non-Linear Mech. 77 (2015) 128-140.

[29] L. Greco, M. Cuomo, An implicit $G^{1}$ multi patch B-spline interpolation for Kirchhoff-Love space rod, Comput. Methods Appl. Mech. Engrg. 269 (2014) 173-197.

[30] L. Greco, M. Cuomo, An isogeometric implicit $G^{1}$ mixed finite element for Kirchhoff space rods, Comput. Methods Appl. Mech. Engrg. 298 (2016) 325-349.

[31] B. Emek Abali, W.H. Müller, V.A. Eremeyev, Strain gradient elasticity with geometric nonlinearities and its computational evaluation, Mech. Adv. Mater. Modern Process. 1 (4) (2015) 1-11.

[32] V.A. Eremeyev, H. Altenbach, in: H. Altenbach, V.A. Eremeyev (Eds.), Basics of Mechanics of Micropolar Shells, in: Shell-like Structures, vol. 572, Springer, Cham, 2017.

[33] E. Turco, Discrete is it enough? The revival of Piola-Hencky keynotes to analyze three-dimensional, Elastica. Contin. Mech. Thermodyn. 30 (5) (2018) 1039-1057.

[34] E. Riks, The application of Newton's method to the problem of elastic stability, J. Appl. Mech. Trans. ASME 39 Ser E (4) (1972) 1060-1065.

[35] O. Rodrigues, Des lois géométriques qui régissent les déplacements d'un système solide dans l'espace, et de la variation des coordonnées provenant de ces déplacements considérés indépendamment des causes qui peuvent les produire, J. Math. Appl. 1 (5) (1840) 380-440.

[36] E. Turco, F. dell'Isola, A. Cazzani, N.L. Rizzi, Hencky-type discrete model for pantographic structures: numerical comparison with second gradient continuum models, Z. Angew. Math. Phys. 67 (85) (2016) 1-28.

[37] P. Wriggers, Nonlinear Finite Element Methods, Springer, 2008.

[38] M.J. Clarke, G.J. Hancock, A study of incremental-iterative strategies for non-linear analyses, Internat. J. Numer. Methods Engrg. 29 (1990) 1365-1391.

[39] G.R. Cowper, The shear coefficient in Timoshenko's beam theory, J. Appl. Mech. 33 (1966) 335-340.

[40] F. dell'Isola, A. Della Corte, A. Battista, E. Barchiesi, Extensible beam models in large deformation under distributed loading: A numerical study on multiplicity of solutions, in: H. Altenbach, W.H. Müller, B. Emek Abali (Eds.), Higher Gradient Materials and Related Generalized Continua, Volume 120, Springer, Cham, 2019.

[41] J. Roorda, A.H. Chilver, Frame buckling: an illustration of the perturbation technique, Int. J. Non-Linear Mech. 5 (1970) 235-246.
[42] M. Pignataro, N. Rizzi, A. Di Carlo, Symmetric bifurcation of plane frames through a modified potential energy approach, J. Struct. Mech. 8 (3) (1980) 237-255.

[43] S.P. Timoshenko, J.M. Gere, Theory of Elastic Stability, second ed., Dover Publications, Mineola, N.Y, 1961.

44] D. Baroudi, I. Giorgio, A. Battista, E. Turco, L.I. Igumnov, Nonlinear dynamics of uniformly loaded elastica: Experimental and numerical evidence of motion around curled stable equilibrium configurations, Z. Angew. Math. Mech. 99 (7) (2019) 1-20.

[45] A. Battista, A. Della Corte, F. dell'Isola, P. Seppecher, Large deformations of 1d microstructured systems modeled as generalized Timoshenko beams, Z. Angew. Math. Phys. 69 (3) (2018) 52.

[46] L. Beirão da Veiga, C. Lovadina, A. Reali, Avoiding shear locking for the Timoshenko beam problem via isogeometric collocation methods, Comput. Methods Appl. Mech. Engrg. 241 (2012) 38-51

[47] F. Auricchio, L. Beirão da Veiga, J. Kiendl, C. Lovadina, A. Reali, Locking-free isogeometric collocation methods for spatial Timoshenko rods, Comput. Methods Appl. Mech. Engrg. 263 (2013) 113-126.

[48] P. Seppecher, J.-J. Alibert, F. dell'Isola, Linear elastic trusses leading to continua with exotic mechanical interactions, J. Phys. Conf. Ser. volume 319 (1) (2011) 012018 .

[49] A. Misra, T. Lekszycki, I. Giorgio, G. Ganzoch, W.H. Müller, F. dell'Isola, Pantographic metamaterials show atypical poynting effect reversal, Mech. Res. Commun. 89 (2018) 6-10.

[50] M. De Angelo, E. Barchiesi, I. Giorgio, B. Emek Abali, Numerical identification of constitutive parameters in reduced order bi-dimensional models for pantographic structures: application to out-of-plane buckling, Arch. Appl. Mech. 89 (7) (2019) 1333-1358.

[51] E. Turco, A. Misra, R. Sarikaya, T. Lekszycki, Quantitative analysis of deformation mechanisms in pantographic substructures: experiments and modeling, Contin. Mech. Thermodyn. 31 (1) (2019) 209-223.

[52] C. Boutin, F. dell'Isola, I. Giorgio, L. Placidi, Linear pantographic sheets. Asymptotic micro-macro models identification, Math. Mech. Complex Syst. 5 (2) (2017) 127-162.

[53] I. Giorgio, F. dell'Isola, D.J. Steigmann, Axisymmetric deformations of a 2nd grade elastic cylinder, Mech. Res. Commun. 94 (2018) 45-48.

[54] I. Giorgio, F. dell'Isola, D.J. Steigmann, Edge effects in hypar nets, C. R. Méc. 347 (2019) 114-123.

[55] E. Barchiesi, G. Ganzosch, C. Liebold, L. Placidi, R. Grygoruk, W.H. Müller, Outof-plane buckling of pantographic fabrics in displacement-controlled shear tests: experimental results and model validation, Contin. Mech. Thermodyn. 31 (2019) $33-45$.

[56] L. Placidi, F. dell'Isola, E. Barchiesi, Heuristic homogenization of Euler and pantographic beams, in: Mechanics of Fibrous Materials and Applications, Springer, Cham, 2020, pp. 123-155

[57] E. Barchiesi, S.R. Eugster, L. Placidi, F. dell'Isola, Pantographic beam: A complete second gradient 1D-continuum in plane, Z. Angew. Math. Phys. 70 (135) (2019).

[58] H. Abdoul-Anziz, P. Seppecher, Strain gradient and generalized continua obtained by homogenizing frame lattices, Math. Mech. Complex Syst. 6 (3) (2018) 213-250.

[59] H. Abdoul-Anziz, P. Seppecher, C. Bellis, Homogenization of frame lattices leading to second gradient models coupling classical strain and strain-gradient terms, Math. Mech. Solids (2019) http://dx.doi.org/10.1177/1081286519855431.

[60] U. Andreaus, M. Spagnuolo, T. Lekszycki, S.R. Eugster, A Ritz approach for the static analysis of planar pantographic structures modeled with nonlinear Euler-Bernoulli beams, Contin. Mech. Thermodyn. (2018) 1-21.

[61] R.W. Ogden, Non-Linear Elastic Deformations, Dover, Mineola, 1997.

[62] Y.B. Fu, R.W. Ogden, Nonlinear stability analysis of pre-stressed elastic bodies, Contin. Mech. Thermodyn. 11 (1999) 141-172.

[63] V.A. Eremeyev, L.M. Zubov, On the stability of elastic bodies with couple stresses, Mech. Solids 29 (3) (1994) 172-181.

[64] D.N. Sheydakov, H. Altenbach, Stability of inhomogeneous micropolar cylindrical tube subject to combined loads, Math. Mech. Solids 21 (9) (2016) 1082-1094.

[65] R.S. Lakes, Stability of Cosserat solids: size effects, ellipticity and waves, J. Mech. Mater. Struct. 13 (1) (2018) 83-91.

[66] Y. Solyaev, S. Lurie, E. Barchiesi, L. Placidi, On the dependence of standard and gradient elastic material constants on a field of defects, Math. Mech. Solids (2019) http://dx.doi.org/10.1177/1081286519861827.

[67] A. Misra, P. Poorsolhjouy, Identification of higher-order elastic constants for grain assemblies based upon granular micromechanics, Math. Mech. Complex Syst. 3 (3) (2015) 285-308.

[68] C. Pideri, P. Seppecher, A second gradient material resulting from the homogenization of an heterogeneous linear elastic medium, Contin. Mech. Thermodyn. 9 (5) (1997) 241-257.

[69] F. dell'Isola, U. Andreaus, L. Placidi, At the origins and in the vanguard of peridynamics, non-local and higher-gradient continuum mechanics: An underestimated and still topical contribution of Gabrio Piola, Math. Mech. Solids 20 (8) (2015) 887-928.

[70] J. Niiranen, V. Balobanov, J. Kiendl, S.B. Hosseini, Variational formulations, model comparisons and numerical methods for Euler-Bernoulli micro- and nano-beam models, Math. Mech. Solids 24 (1) (2019) 312-335. 
[71] I. Giorgio, D. Del Vescovo, Energy-based trajectory tracking and vibration control for multi-link highly flexible manipulators, Math. Mech. Complex Syst. 7 (2) (2019) 159-174.

[72] I. Giorgio, D. Del Vescovo, Non-linear lumped-parameter modeling of planar multi-link manipulators with highly flexible arms, Robotics 7 (4) (2018).

[73] I. Giorgio, A. Della Corte, F. dell'Isola, Dynamics of 1D nonlinear pantographic continua, Nonlinear Dynam. 88 (1) (2017) 21-31.

[74] J. Chróścielewski, R. Schmidt, V.A. Eremeyev, Nonlinear finite element modeling of vibration control of plane rod-type structural members with integrated piezoelectric patches, Contin. Mech. Thermodyn. 31 (1) (2019) 147-188.
[75] L. Greco, M. Cuomo, L. Contrafatto, A reconstructed local $\bar{B}$ formulation for isogeometric Kirchhoff-Love shells, Comput. Methods Appl. Mech. Engrg. 332 (2018) 462-487.

[76] L. Greco, M. Cuomo, L. Contrafatto, A quadrilateral G1-conforming finite element for the Kirchhoff plate model, Comput. Methods Appl. Mech. Engrg. 346 (2019) 913-951.

[77] L. Greco, M. Cuomo, L. Contrafatto, Two new triangular G1-conforming finite elements with cubic edge rotation for the analysis of Kirchhoff plates, Comput. Methods Appl. Mech. Engrg. 356 (2019) 354-386.

[78] S.T. Yaghoubi, V. Balobanov, S.M. Mousavi, J. Niiranen, Variational formulations and isogeometric analysis for the dynamics of anisotropic gradient-elastic EulerBernoulli and shear-deformable beams, Eur. J. Mech. A Solids 69 (2018) $113-123$. 